\title{
Piecewise linear approximation of fuzzy numbers: algorithms, arithmetic operations and stability of characteristics
}

\author{
Lucian Coroianu ${ }^{1} \cdot$ Marek Gagolewski ${ }^{2,3} \cdot$ Przemyslaw Grzegorzewski ${ }^{2,3}$ (D)
}

Published online: 14 February 2019

(c) The Author(s) 2019

\begin{abstract}
The problem of the piecewise linear approximation of fuzzy numbers giving outputs nearest to the inputs with respect to the Euclidean metric is discussed. The results given in Coroianu et al. (Fuzzy Sets Syst 233:26-51, 2013) for the 1-knot fuzzy numbers are generalized for arbitrary $n$-knot $(n \geq 2)$ piecewise linear fuzzy numbers. Some results on the existence and properties of the approximation operator are proved. Then, the stability of some fuzzy number characteristics under approximation as the number of knots tends to infinity is considered. Finally, a simulation study concerning the computer implementations of arithmetic operations on fuzzy numbers is provided. Suggested concepts are illustrated by examples and algorithms ready for the practical use. This way, we throw a bridge between theory and applications as the latter ones are so desired in real-world problems.
\end{abstract}

Keywords Approximation of fuzzy numbers - Calculations on fuzzy numbers - Characteristics of fuzzy numbers . Fuzzy number · Piecewise linear approximation

\section{Introduction}

A family of fuzzy numbers constitutes an important subclass of fuzzy sets having countless applications in all cases where imprecise real values are modeled by their fuzzy counterparts. To avoid problems in processing and calculations on fuzzy numbers described by complicated membership functions the suitable approximations are commonly applied. In particular, the interval (see Chanas 2001; Grzegorzewski 2002, 2012), triangular (see Abbasbandy et al. 2010; Ban 2011; Ban and Coroianu 2015a, 2016; Yeh 2017) or trapezoidal approximation (see Abbasbandy and Amirfakhrian 2006; Abbasbandy and Asady 2004; Abbasbandy and Hajjari 2009b; Ban 2008, 2009a,b; Ban et al. 2011a,b; Ban

Communicated by A. Di Nola.

Przemyslaw Grzegorzewski

pgrzeg@ibspan.waw.pl

1 Department of Mathematics and Informatics, University of Oradea, 1 Universitatii Street, 410087 Oradea, Romania

2 Faculty of Mathematics and Information Science, Warsaw University of Technology, Koszykowa 75, 00-662 Warsaw, Poland

3 Systems Research Institute, Polish Academy of Sciences, Newelska 6, 01-447 Warsaw, Poland and Coroianu 2011, 2012, 2014; Coroianu 2011, 2012; Grzegorzewski 2008a,b, 2010; Grzegorzewski and Mrówka 2005, 2007; Grzegorzewski and Pasternak-Winiarska 2009, 2014; Yeh 2007, 2008a, b) is very popular, mainly because of simplicity of the output representation (by no more than four points). More recently (see Ban et al. 2016; Yeh and Chu 2011), the studies were extended by employing approximations by L-R fuzzy numbers. Fuzzy number approximation via shadowed sets was discussed in Grzegorzewski (2013). For the review of the fuzzy numbers approximation methods and their applications, we refer the reader to the monograph Ban et al. (2015).

One of the reasons that the trapezoidal fuzzy numbers are so popular in applications is that each such fuzzy number is represented completely by four real numbers only. Of course, the representation simplicity is a strong advantage but one may consider whether the shape reduction going so far is not too impoverish and restrictive in some situations. Therefore, the problem of the piecewise linear approximation of fuzzy numbers by the so-called 1-knot fuzzy numbers was considered in Coroianu et al. (2013). Each such 1-knot fuzzy number is completely characterized by six points on the real line. This way we get fuzzy numbers which are still simple enough, but simultaneously, having more "degrees of freedom", we may obtain approximations that are more flex- 
ible to preserve some important properties of the input fuzzy numbers.

In this paper, a generalization of the results presented in Coroianu et al. (2013) is given. Now, instead of six points on the real line and piecewise linear sides each consisting of two segments that characterize 1-knot fuzzy numbers, we consider $n$-knot fuzzy numbers (where $n \geq 2$ ) which enables to quantify the uncertainty at $n$ intermediate levels between 0 and 1. Such fuzzy numbers were already introduced in paper Báez-Sánchez et al. (2012) and were called polygonal fuzzy numbers. In this way, we obtain a subfamily of fuzzy numbers with piecewise linear membership functions, where each side consists of $n+1$ segments. Hence, the output of the approximation is still simple but more flexible for reconstructing a fuzzy input than obtained using other methods discussed above. Moreover, it turns out that basic characteristics of the approximations converge to corresponding characteristics of the input fuzzy numbers. When comparing $n$-knot approximation with the 1-knot, discussed in Coroianu et al. (2013), its natural disadvantage is the longer processing time caused by more knots but its obvious advantage is better accuracy.

It should be stressed that the $n$-knot fuzzy number is the most natural and desired fuzzy structure for the computer representation which is by definition discrete, even if the original object it represents is a continuous one. Actually, a typical way a fuzzy object is stored in a database is a set of its $\alpha$-cuts. Here a natural question arises about the adequate choice of those $\alpha$-cuts, even for a fixed set of $\alpha$-levels. The method of the nearest piecewise linear approximation of fuzzy numbers proposed in this paper enables to avoid unjustified subjectivity and to choose the required $\alpha$-cuts in an objective way based on the nearness criteria crucial to any reasonable approximation.

The paper is organized as follows. In Sect. 2, we recall basic terminology connected with fuzzy numbers and define the $\alpha$-piecewise linear $n$-knot fuzzy numbers. In Sect. 3, we introduce some auxiliary results and present a convenient reparametrization of piecewise linear fuzzy numbers useful for solving the approximation problem. Then in Sect. 4, we discuss briefly the existence and properties of the nearest piecewise linear fuzzy number for the Euclidean distance and a fixed knot setting.

Next section, i.e., Sect. 5, contains a broad study on the convergence results concerning the approximation operator. In particular, we consider some special cases, like the socalled naïve approximator (i.e., an $n$-knot piecewise linear fuzzy number that interpolates the sides of a fuzzy number at the knots) or the approximator with equidistant knots. We prove there some theorems on the rate of convergence but we also examine the stability of basic characteristics like the expected interval, expected value, value of fuzzy number and its ambiguity.
Section 6 is both theoretical and strongly user-oriented as well. We give there not only practical approximation algorithms and illustrative examples but we also provide a simulation study on the approximation accuracy of the computer calculations on fuzzy numbers and stability of some fuzzy number characteristics (for the practical implementation of the presented algorithms we refer the reader to FuzzyNumbers package for R by Gagolewski 2015). Therefore, Sect. 6 appears in some sense as the core of the paper by linking theory with applications as the latter ones are so desired in real-world problems.

Finally, Sect. 7 concludes the paper. Some open problems and directions for the further research are also sketched there.

\section{Piecewise linear fuzzy numbers}

Fuzzy numbers are particular cases of convex fuzzy sets on the real line. The membership function of a fuzzy number $A$ is given by

$A(x)= \begin{cases}0 & \text { if } x<a_{1}, \\ l_{A}(x) & \text { if } a_{1} \leq x<a_{2}, \\ 1 & \text { if } a_{2} \leq x \leq a_{3}, \\ r_{A}(x) & \text { if } a_{3}<x \leq a_{4}, \\ 0 & \text { if } x>a_{4},\end{cases}$

where $a_{1}, a_{2}, a_{3}, a_{4} \in \mathbb{R}, l_{A}:\left[a_{1}, a_{2}\right] \longrightarrow[0,1]$ is a nondecreasing upper semicontinuous function, $l_{A}\left(a_{1}\right)=0$, $l_{A}\left(a_{2}\right)=1$, called the left side of the fuzzy number, and $r_{A}:\left[a_{3}, a_{4}\right] \longrightarrow[0,1]$ is a nonincreasing upper semicontinuous function, $r_{A}\left(a_{3}\right)=1, r_{A}\left(a_{4}\right)=0$, called the right side of the fuzzy number $A$. The $\alpha$-cut of $A, \alpha \in(0,1]$, is a crisp set defined as

$A_{\alpha}=\{x \in \mathbb{R}: A(x) \geq \alpha\}$.

The case of the 0 -cut is defined in slightly different way, and it actually coincides with the support of the fuzzy number. More exactly we have

$\operatorname{supp}(A)=A_{0}=\overline{\{x \in \mathbb{R}: A(x)>0\}}$.

From the convexity property, it easily results that every $\alpha$-cut of a fuzzy number is a compact interval

$A_{\alpha}=\left[A_{L}(\alpha), A_{U}(\alpha)\right]$,

where $A_{L}(\alpha)=\inf \{x \in \mathbb{R}: A(x) \geq \alpha\}$ and $A_{U}(\alpha)=$ $\sup \{x \in \mathbb{R}: A(x) \geq \alpha\}$.

The 1-cut of $A$ is also called the core of $A$ and the following notation is commonly used

$A_{1}=\operatorname{core}(A)=\{x \in \mathbb{R}: A(x)=1\}$. 
When $\operatorname{core}(A)$ is reduced to a single element, then $A$ is called a unimodal fuzzy number [in some works like the book Hanss (2005), the authors reduce the term fuzzy numbers to unimodal fuzzy numbers only and call multimodal fuzzy numbers as fuzzy intervals]. Further on we denote by $\mathbb{F}(\mathbb{R})$ the set of all fuzzy numbers.

However, fuzzy numbers with simple membership functions are often preferred in practice. For example, triangular or trapezoidal fuzzy numbers are most often used to rank fuzzy numbers (see, e.g., Abbasbandy and Hajjari 2009a; Ban and Coroianu 2015b; Facchinetti and Ricci 2004). Then, the same classes or even more generally the well-known class of L-R fuzzy numbers are used in fuzzy arithmetic (see, e.g., Carlsson and Fullér 2011; Hanss 2005; Hong and Hwang 1997; Kolesárová 1995). We can also mention here the Bodjanova (2005) fuzzy numbers used in statistical problems or in multicriteria decision making (see Ban and Ban 2012). Finally, we mention the recently introduced so-called parametric fuzzy numbers also known as semi-trapezoidal fuzzy numbers (see Nasibov and Peker 2008; Yeh 2009, 2011). Apart from the aforementioned applications, such fuzzy numbers are very suitable in approximation as we already discussed this in Introduction. Another subclass of $\mathbb{F}(\mathbb{R})$, very useful and convenient especially in computer processing, may be defined by considering fuzzy numbers with piecewise linear sides. We consider a particular type of such fuzzy numbers introduced in Báez-Sánchez et al. (2012) and being referred there as polygonal fuzzy numbers. Consider the following definition.

Definition 1 (see Definition 5 and Example 6 in BáezSánchez et al. 2012) Fix $n \in \mathbb{N}_{0}$, and let $\mathfrak{A}_{n}=\left\{\left(\alpha_{0}, \alpha_{1}, \ldots\right.\right.$, $\left.\alpha_{n+1}\right) \in[0,1]^{n+2}: 0=\alpha_{0}<\alpha_{1}<\cdots<\alpha_{n}<\alpha_{n+1}=$ $1)\}$, and $\mathfrak{S}_{n}=\left\{\left(s_{1}, \ldots, s_{2 n+4}\right) \in \mathbb{R}^{2 n+4}: s_{1} \leq \cdots \leq\right.$ $\left.s_{2 n+4}\right\}$. Given $\boldsymbol{\alpha} \in \mathfrak{A}_{n}$ and $\mathbf{s} \in \mathfrak{S}_{n}$, an $\boldsymbol{\alpha}$-piecewise linear $n$ knot fuzzy number (also known as polygonal fuzzy number according to Báez-Sánchez et al. 2012) $S(\boldsymbol{\alpha}, \mathbf{s})$ is defined by:

$$
\begin{gathered}
S(\boldsymbol{\alpha}, \mathbf{s})_{L}(\beta)=s_{i+1}+\left(s_{i+2}-s_{i+1}\right) \\
\frac{\beta-\alpha_{i}}{\alpha_{i+1}-\alpha_{i}}, \\
S(\boldsymbol{\alpha}, \mathbf{s})_{U}(\beta)=s_{2 n+4-i}+\left(s_{2 n+3-i}-s_{2 n+4-i}\right) \\
\frac{\beta-\alpha_{i}}{\alpha_{i+1}-\alpha_{i}},
\end{gathered}
$$

for some $i \in\{0, \ldots, n\}$ such that $\beta \in\left[\alpha_{i}, \alpha_{i+1}\right]$.

Please note that by the connection between the functions $l_{S(\boldsymbol{\alpha}, \mathbf{s})}, r_{S(\boldsymbol{\alpha}, \mathbf{s})}$ and $S(\boldsymbol{\alpha}, \mathbf{s})_{L}, S(\boldsymbol{\alpha}, \mathbf{s})_{U}$, the membership function of $S(\boldsymbol{\alpha}, \mathbf{s})$ is also piecewise linear in the case when $\mathbf{s}$ is strictly monotone (as an example see Fig. 1).

Let $\mathbb{F}^{\pi_{n}(\boldsymbol{\alpha})}(\mathbb{R})$ denote the set of all $\boldsymbol{\alpha}$-piecewise linear $n$ knot fuzzy numbers (for fixed $n$ and $\boldsymbol{\alpha}$ ). It is worth noting

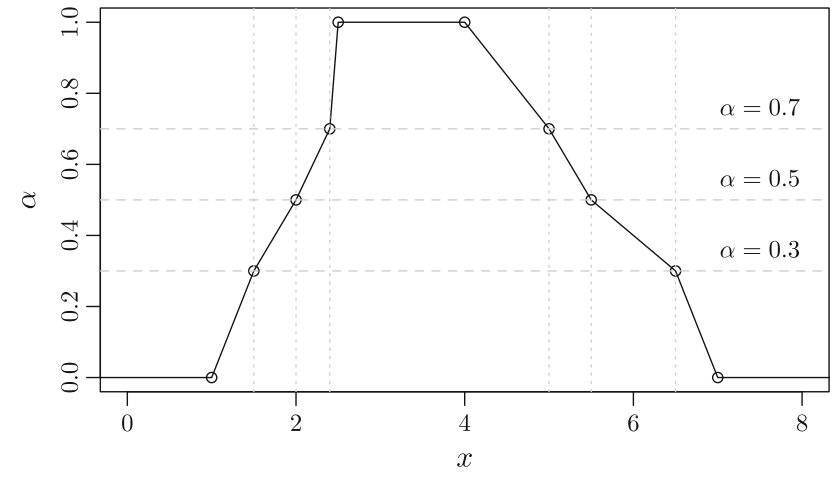

Fig. 1 Plot of an exemplary 3-knot piecewise linear fuzzy number $S(\boldsymbol{\alpha}, \mathbf{s})$, with $\boldsymbol{\alpha}=(0.3,0.5,0.7)$ and $\mathbf{s}=$ $(1,1.5,2,2.4,2.5,4,5,5.5,6.5,7)$

that the class introduced in Definition 1 generalizes wellknown subfamilies of fuzzy numbers. Actually, for $n=0$ and $s_{1}=s_{4}$ we get "crisp" real numbers, for $n=0$ and $s_{1}=s_{2}, s_{3}=s_{4}$ we obtain "crisp" real intervals; if $n=0$ and $s_{2}=s_{3}$, we get triangular fuzzy numbers, assuming only $n=0$ we obtain trapezoidal fuzzy numbers, while for $n=1$ we receive 1-knot piecewise linear fuzzy numbers, discussed in Coroianu et al. (2013).

Further on we assume that two fuzzy numbers $A$ and $B$ are equal (and denote it as $A=B$ ) if $A_{L}(\beta)=B_{L}(\beta)$ and $A_{U}(\beta)=B_{U}(\beta)$ almost everywhere, $\beta \in[0,1]$.

Let $A, B \in \mathbf{F}(\mathbb{R})$, and $\lambda \in \mathbb{R}$. We define the sum $A+B$ and the scalar multiplication $\lambda \cdot A$ (see, e.g., Diamond and Kloeden 1994, p. 40) for every $\beta \in[0,1]$ as:

$$
\begin{aligned}
(A+B)_{\beta} & =A_{\beta}+B_{\beta} \\
& =\left[A_{L}(\beta)+B_{L}(\beta), A_{U}(\beta)+B_{U}(\beta)\right]
\end{aligned}
$$

and

$$
(\lambda \cdot A)_{\beta}=\lambda A_{\beta}= \begin{cases}{\left[\lambda A_{L}(\beta), \lambda A_{U}(\beta)\right]} & \text { if } \lambda \geq 0 \\ {\left[\lambda A_{U}(\beta), \lambda A_{L}(\beta)\right]} & \text { if } \lambda<0 .\end{cases}
$$

In the case of $\alpha$-piecewise linear $n$-knot fuzzy numbers $S^{\prime}=S\left(\boldsymbol{\alpha}, \mathbf{s}^{\prime}\right)$ and $S^{\prime \prime}=S\left(\boldsymbol{\alpha}, \mathbf{s}^{\prime \prime}\right)$, we obtain

$S^{\prime}+S^{\prime \prime}=S\left(\boldsymbol{\alpha}, \mathbf{s}^{\prime}+\mathbf{s}^{\prime \prime}\right)$.

Moreover, we have

$$
\lambda S= \begin{cases}S(\boldsymbol{\alpha}, \lambda \mathbf{s}) & \text { if } \lambda \geq 0 \\ S\left(\boldsymbol{\alpha}, \lambda\left(s_{2 n+4}, s_{2 n+3}, \ldots, s_{1}\right)\right) & \text { if } \lambda<0 .\end{cases}
$$

Thus, $\mathbb{F}^{\pi_{n}(\boldsymbol{\alpha})}(\mathbb{R})$ is closed under these two extension principle-based arithmetic operations. 


\section{Some auxiliary results}

Many papers show that the most suitable and commonly used metric for fuzzy numbers approximation is an extension of the Euclidean distance $d$ defined by (see Grzegorzewski 1998)

$$
\begin{aligned}
d^{2}(A, B)= & \int_{0}^{1}\left(A_{L}(\beta)-B_{L}(\beta)\right)^{2} \mathrm{~d} \beta \\
& +\int_{0}^{1}\left(A_{U}(\beta)-B_{U}(\beta)\right)^{2} \mathrm{~d} \beta .
\end{aligned}
$$

Let $L^{2}[0,1]$ denote the space of square integrable functions on $[0,1]$. It is well known (cf. Yeh 2011) that we can embed the space $(\mathbb{F}(\mathbb{R}), d,+, \cdot)$ into the Hilbert space $\left(L^{2}[0,1] \times L^{2}[0,1], \widetilde{d}, \oplus, \odot\right)$, such that for $\mathbf{f}=\left(f_{1}, f_{2}\right)$, $\mathbf{g}=\left(g_{1}, g_{2}\right) \in L^{2}[0,1] \times L^{2}[0,1]$ we have

$$
\begin{aligned}
\widetilde{d}^{2}(\mathbf{f}, \mathbf{g})= & \int_{0}^{1}\left(f_{1}(\alpha)-g_{1}(\alpha)\right)^{2} \mathrm{~d} \alpha \\
& +\int_{0}^{1}\left(f_{2}(\alpha)-g_{2}(\alpha)\right)^{2} \mathrm{~d} \alpha,
\end{aligned}
$$

and

$A \oplus B=A+B$,

$\lambda \odot A=\lambda \cdot A$

for any $A, B \in \mathbb{F}(\mathbb{R})$ and $\lambda \in[0, \infty)$, as $\left(A_{L}, A_{U}\right)$, $\left(B_{L}, B_{U}\right) \in L^{2}[0,1] \times L^{2}[0,1]$ (for the proof see, e.g., Yeh 2011). Because there is no risk of confusion, in all what follows, we use + and $\cdot$ to define the addition and scalar multiplication in the space $L^{2}[0,1] \times L^{2}[0,1]$, instead of $\oplus$ and $\odot$.

Note that the inner product which generates $\widetilde{d}$ is given by (see, e.g., Yeh 2008a)

$\langle\mathbf{f}, \mathbf{g}\rangle=\int_{0}^{1} f_{1}(\alpha) g_{1}(\alpha) \mathrm{d} \alpha+\int_{0}^{1} f_{2}(\alpha) g_{2}(\alpha) \mathrm{d} \alpha$.

Assuming a fixed $n$ and a fixed family of $\alpha$-cuts $\alpha=$ $\left(\alpha_{0}, \ldots, \alpha_{n+1}\right) \in \mathfrak{A}_{n}$, let us define the following set of vectors $\mathbf{e}_{1}, \ldots, \mathbf{e}_{2 n+4} \in L^{2}[0,1] \times L^{2}[0,1], \mathbf{e}_{i}=\left(e_{i, 1}, e_{i, 2}\right)$, $i=1, \ldots, 2 n+4$, such that for $\beta \in[0,1]$ :

$-e_{1,1}(\beta)=e_{1,2}(\beta)=1$,

- for $i=2, \ldots, n+2$ :

$$
e_{i, 1}(\beta)= \begin{cases}0 & \text { for } \beta<\alpha_{i-2}, \\ \frac{\beta-\alpha_{i-2}}{\alpha_{i-1}-\alpha_{i-2}} & \text { for } \beta \in\left[\alpha_{i-2}, \alpha_{i-1}\right], \\ 1 & \text { for } \beta>\alpha_{i-1},\end{cases}
$$$$
e_{i, 2}(\beta)=1 \text {, }
$$

$$
\begin{aligned}
& -e_{n+3,1}(\beta)=0, e_{n+3,2}(\beta)=1 \text {, } \\
& \text { - for } i=n+4, \ldots, 2 n+4 \text { : } \\
& e_{i, 1}(\beta)=0 \text {, } \\
& e_{i, 2}(\beta)=\left\{\begin{array}{lr}
1 & \text { for } \beta<\alpha_{2 n-i+4}, \\
\frac{\alpha_{2 n-i+5}-\beta}{\alpha_{2 n-i+5}-\alpha_{2 n-i+4}} & \text { for } \beta \in\left[\alpha_{2 n-i+4},\right. \\
0 & \left.\alpha_{2 n-i+5}\right], \\
0 & \text { for } \beta>\alpha_{2 n-i+5},
\end{array}\right.
\end{aligned}
$$

The set of vectors defined above is extremely useful for a reparametrization of piecewise linear fuzzy numbers, which appears very convenient in further considerations. Namely, given any piecewise linear $n$-knot fuzzy number $S=\mathrm{S}(\boldsymbol{\alpha}, \mathbf{s})$, let $\delta=\left(\delta_{1}, \ldots, \delta_{2 n+4}\right)$ with $\delta_{1}=s_{1}$ and $\delta_{i}:=s_{i}-s_{i-1}$ for $i=2, \ldots, 2 n+4$. It can be seen that we have $S=$ $\sum_{i=1}^{2 n+4} \delta_{i} \mathbf{e}_{i}$. From now on we use the notation $\mathrm{S}_{d}(\boldsymbol{\alpha}, \boldsymbol{\delta})$ to represent $S$ in its new parametrization.

Lemma 1 The set $\left\{\mathbf{e}_{1}, \mathbf{e}_{2}, \ldots, \mathbf{e}_{2 n+4}\right\}$ is linearly independent in $L^{2}[0,1] \times L^{2}[0,1]$.

Proof First of all let us note that we have

$\mathbb{F}^{\pi_{n}(\alpha)}(\mathbb{R})=\left\{\sum_{i=1}^{2 n+4} \delta_{i} \mathbf{e}_{i}: \delta_{1} \in \mathbb{R}, \delta_{2}, \ldots, \delta_{2 n+4} \in[0, \infty)\right\}$

Therefore, to obtain the desired conclusion it suffices to find $2 n+4$ vectors in $\mathbb{F}^{\pi_{n}(\alpha)}(\mathbb{R})$ which are linearly independent in $L^{2}[0,1] \times L^{2}[0,1]$. Obviously such vectors exist as for example $\mathrm{S}_{d}\left(\alpha, \xi_{i}\right), i=1, \ldots, 2 n+4$, where $\left\{\xi_{1}, \ldots, \xi_{2 n+4}\right\}$ is the canonical base of $\mathbb{R}^{2 n+4}$.

Now we are in position to present the main result of this section.

Lemma 2 For any $\boldsymbol{\alpha} \in \mathfrak{A}_{n}$ the set $\mathbb{F}^{\pi_{n}(\boldsymbol{\alpha})}(\mathbb{R})$ is a closed convex subset of the space $L^{2}[0,1] \times L^{2}[0,1]$ endowed with the topology generated by metric $\widetilde{d}$.

Proof If we denote $\mathbb{V}=\operatorname{span}\left\{\mathbf{e}_{1}, \mathbf{e}_{2}, \ldots, \mathbf{e}_{2 n+4}\right\}$ then let us consider the linear bijective transformation $i: \mathbb{R}^{2 n+4} \rightarrow \mathbb{V}$, $i\left(x_{1}, x_{2}, \ldots, x_{2 n+4}\right)=\sum_{i=1}^{2 n+4} x_{i} \mathbf{e}_{i}$. We consider on $\mathbb{V}$ the same metric $d$ as on $L^{2}[0,1] \times L^{2}[0,1]$ (hence by (6) $\mathbb{V}$ is an inner product space) and $\mathbb{R}^{2 n+4}$ is endowed with the Euclidean topology. Since $i$ and $i^{-1}$ are linear transformations between finite-dimensional normed spaces, it is immediate that both are continuous and hence homeomorphisms. We observe that $\mathbb{F}^{\pi_{n}(\boldsymbol{\alpha})}(\mathbb{R})=i(\Omega)$ where $\Omega=$ $\left\{\left(x_{1}, \ldots, x_{2 n+4}\right) \in \mathbb{R}^{2 n+4}: x_{i} \geq 0, i=2, \ldots, 2 n+4\right\}$. Obviously $\Omega$ is closed in $\mathbb{R}^{2 n+4}$ (actually $\Omega$ is a polyhedral subset of $\mathbb{R}^{2 n+4}$ and all such sets are closed in $\mathbb{R}^{2 n+4}$ ) and since $i^{-1}$ is continuous it results that $\mathbb{F}^{\pi_{n}(\boldsymbol{\alpha})}(\mathbb{R})=i(\Omega)$ is 
a closed subset of $\mathbb{V}$. On the other hand, $\mathbb{V}$ is a finite linear subspace of $L^{2}[0,1] \times L^{2}[0,1]$ and hence $\mathbb{V}$ is closed in $L^{2}[0,1] \times L^{2}[0,1]$. By elementary topology, it easily results now that $\mathbb{F}^{\pi_{n}(\boldsymbol{\alpha})}(\mathbb{R})$ is closed in $L^{2}[0,1] \times L^{2}[0,1]$.

As the convexity of $\mathbb{F}^{\pi_{n}(\boldsymbol{\alpha})}(\mathbb{R})$ is obvious, the proof is complete.

Remark 1 From the above proof, it follows that a sequence of $\boldsymbol{\alpha}$-piecewise linear $n$-knot fuzzy numbers, $\left(\mathrm{S}_{d}\left(\boldsymbol{\alpha}, \boldsymbol{\delta}_{m}\right)\right)_{m=1,2, \ldots}$ with $\boldsymbol{\delta}_{m}=\left(\delta_{m, i}\right)_{i=1, \ldots, 2 n+4}$, converges to $S_{d}(\boldsymbol{\alpha}, \boldsymbol{\delta})$, where $\boldsymbol{\delta}=\left(\delta_{i}\right)_{i=1, \ldots, 2 n+4}$ if and only if for any $i$ we have $\delta_{m, i} \rightarrow \delta_{i}$, which is equivalent with $\left\|\boldsymbol{\delta}_{m}-\boldsymbol{\delta}\right\| \rightarrow 0$ (here $\|\cdot\|$ denotes the Euclidean norm over the space $\mathbb{R}^{2 n+4}$ ).

Remark 2 Lemma 2 can be deduced from Corollary 12 in Báez-Sánchez et al. (2012). Although in Báez-Sánchez et al. (2012), a different metric is employed one can easily prove that this metric is equivalent with the Euclidean metric on the span of $\mathbb{F}^{\pi_{n}(\boldsymbol{\alpha})}(\mathbb{R})$. However, we prefer the present proof because it is more suitable to our forthcoming results. Especially, we refer here to the set $\left\{\mathbf{e}_{1}, \mathbf{e}_{2}, \ldots, \mathbf{e}_{2 n+4}\right\}$ of linearly independent vectors in $L^{2}[0,1] \times L^{2}[0,1]$ which will be used to approach the main results of the paper.

\section{The best approximation for fixed $\alpha$}

In this section, we generalize the theoretical results from paper Coroianu et al. (2013) concerning the existence, uniqueness and characterization of the approximation as well as the properties of the derived approximation operator by extending them to the case of piecewise linear $n$-knot fuzzy numbers.

If membership functions of fuzzy numbers under study are too complicated, we usually approximate them by some simpler forms that are more useful for processing and easier to interpret. In this section, we discuss how to approximate an arbitrary fuzzy number by a piecewise linear $n$-knot fuzzy number described by fixed $\alpha$-cuts.

Hence, given a fuzzy number $A \in \mathbb{F}(\mathbb{R})$, fixed $n$, and a fixed family of $\alpha$-cuts $\boldsymbol{\alpha} \in \mathfrak{A}_{n}$, we are interested in finding $S_{\alpha}^{*}(A) \in \mathbb{F}^{\pi_{n}(\alpha)}(\mathbb{R})$ such that:

$d\left(A, S_{\alpha}^{*}(A)\right)=\min _{S \in \mathbb{F}^{\pi_{n}(\alpha)}(\mathbb{R})} d(A, S)$.

Using similar reasoning as in paper Coroianu et al. (2013), one can prove that the above problem has a unique solution. By showing this fact, we are able to define the approximation operator $\Pi_{\alpha}^{n}: \mathbb{F}(\mathbb{R}) \rightarrow \mathbb{F}^{\pi_{n}(\boldsymbol{\alpha})}(\mathbb{R})$ which assigns to a fuzzy number $A$ its unique best piecewise linear approximator (for given $n$ and $\boldsymbol{\alpha}$ ).
Theorem 1 If $A$ is an arbitrary fuzzy number, then there exists the unique fuzzy number $\Pi_{\alpha}^{n}(A) \in \mathbb{F}^{\pi_{n}(\boldsymbol{\alpha})}(\mathbb{R})$ satisfying (8).

Taking into account Lemma 2, we obtain the proof (we omit this proof since it is identical with the proof of Theorem 8 in Coroianu et al. 2013).

Now let us denote with $\Phi=\left(\phi_{i, j}\right)_{i, j=1, \ldots, 2 n+4}$, the Gram matrix associated with the set $\left\{\mathbf{e}_{1}, \ldots, \mathbf{e}_{2 n+4}\right\}$, i.e., $\phi_{i, j}=\left\langle\mathbf{e}_{i}, \mathbf{e}_{j}\right\rangle$. Since these vectors are linearly independent, it follows that $\Phi$ is invertible. Moreover, for fixed $A$ let $\mathbf{b}=\left(b_{1}, \ldots, b_{2 n+4}\right)$ such that $b_{i}=\left\langle A, \mathbf{e}_{i}\right\rangle$.

We have the following characterization of the best approximation (see, e.g., Yeh 2009, Fact 2.1). If $(X,\langle\cdot, \cdot\rangle)$ is a Hilbert space, $\Omega$ is a closed convex subset of $X$, and $x \in X$, then $x^{*} \in \Omega$ is the unique best approximation of $x$ relatively to the set $\Omega$ if and only if $\left\langle x-x^{*}, y-x^{*}\right\rangle \leq 0$ for any $y \in \Omega$. Note that the notation $x^{*}=P_{\Omega}(x)$ is often used to denote that $x^{*}$ is the projection of $x$ onto $\Omega$.

Theorem 2 Let $\alpha \in \mathfrak{A}_{n}$ be fixed and let $A$ denote a fuzzy number. Then $\Pi_{\boldsymbol{\alpha}}^{n}(A)=\mathrm{S}_{d}\left(\boldsymbol{\alpha}, \boldsymbol{\delta}^{*}\right), \boldsymbol{\delta}^{*} \in \mathbb{R} \times \mathbb{R}_{+}^{2 n+3}$, is the unique best approximation of $A$ relatively to the set $\mathbb{F}^{\pi_{n}(\boldsymbol{\alpha})}(\mathbb{R})$ with respect to the Euclidean metric d, if and only if there exists a vector $\mathbf{z}^{*} \in \mathbb{R}^{2 n+4}$ such that all the following requirements hold

(i) $\Phi \boldsymbol{\delta}^{* T}-\mathbf{z}^{* T}=\mathbf{b}^{T}$,

(ii) $z_{1}^{*}=0$ and $z_{i}^{*} \geq 0, \forall i>1$,

(iii) $\delta_{i}^{*}=0$ or $z_{i}^{*}=0, \forall i>1$.

We omit the proof because it uses a similar reasoning as the proof of Theorem 9 in Coroianu et al. (2013). Basically the only difference is that now we have a Gram matrix of dimension $2 n+4$.

It turns out that our approximation operator has some very important properties from the well-known list presented in Grzegorzewski and Mrówka (2005).

Theorem 3 For any $\alpha \in \mathfrak{A}_{n}$, the $\Pi_{\alpha}^{n}$ operator fulfills the following properties:

(i) Identity, i.e., $\Pi_{\alpha}^{n}(A)=A, \forall A \in \mathbb{F}^{\pi_{n}(\alpha)}(\mathbb{R})$;

(ii) Invariance to translation, i.e., $\Pi_{\alpha}^{n}(A+z)=\Pi_{\alpha}^{n}(A)+z, \forall A \in \mathbb{F}(\mathbb{R}), \forall z \in \mathbb{R} ;$

(iii) Scale invariance, i.e., $\Pi_{\alpha}^{n}(\lambda A)=\lambda \Pi_{\alpha}^{n}(A) f, \forall A \in \mathbb{F}(\mathbb{R}), \forall \lambda \in \mathbb{R} ;$

(iv) Lipschitz-continuity, i.e., $d\left(\Pi_{\alpha}^{n}(A), \Pi_{\alpha}^{n}(B)\right) \leq d(A, B), \forall A, B \in \mathbb{F}(\mathbb{R})$.

The proofs are identical with those from the particular case of piecewise linear 1-knot approximation (see Theorem 10 in Coroianu et al. 2013). 


\section{Some remarks on convergence}

For the piecewise linear approximation operator introduced in the previous section, we can prove useful approximation results. First of all we can find a rate of convergence for the sequence of piecewise linear approximations by letting $n \rightarrow \infty$. Additionally, we can estimate the convergence rate in the approximation of important characteristics associated to fuzzy numbers such as the value, ambiguity and expected interval, respectively. The key element in the process of obtaining the convergence results is the use of the well-known naïve approximator.

\subsection{The naïve approximator}

In what follows, we propose some convergence results for sequences of piecewise approximations. However, firstly, for given fuzzy number $A, n \in \mathbb{N}$, and $\boldsymbol{\alpha}_{n}=\left(\alpha_{n, 0}, \alpha_{n, 1}, \ldots\right.$, $\left.\alpha_{n, n+1}\right) \in \mathfrak{A}_{n}$, let us consider a fuzzy number $N_{\boldsymbol{\alpha}_{n}}^{n}(A) \in$ $\mathbb{F}^{\pi_{n}(\boldsymbol{\alpha})}(\mathbb{R})$ defined by

$$
\begin{aligned}
\left(N_{\boldsymbol{\alpha}_{n}}^{n}(A)\right)_{L}(\beta)= & A_{L}\left(\alpha_{n, i}\right) \\
& +\frac{A_{L}\left(\alpha_{n, i+1}\right)-A_{L}\left(\alpha_{n, i}\right)}{\alpha_{n, i+1}-\alpha_{n, i}}\left(\beta-\alpha_{n, i}\right), \\
\left(N_{\boldsymbol{\alpha}_{n}}^{n}(A)\right)_{U}(\beta)= & A_{U}\left(\alpha_{n, i}\right) \\
& +\frac{A_{U}\left(\alpha_{n, i+1}\right)-A_{U}\left(\alpha_{n, i}\right)}{\alpha_{n, i+1}-\alpha_{n, i}}\left(\beta-\alpha_{n, i}\right)
\end{aligned}
$$

for $i=0, \ldots, n$ and $\beta \in\left[\alpha_{i}, \alpha_{i+1}\right]$. This is the so-called naïve approximator of $A$, i.e., an $n$-knot piecewise linear fuzzy number that interpolates both $A_{L}$ and $A_{U}$ at the knots (it minimizes the $L_{2}$ metric exactly at finite number of points, while the approximator suggested in this paper minimizes the metric "globally", i.e., at all alpha cuts). This operator was also proposed in Báez-Sánchez et al. (2012) where it was used as an approximator with respect to the Hausdorff metric. We will use this operator to obtain approximation results for the sequence $\left(\Pi_{\alpha_{n}}^{n}\right)_{n>1}$ with respect to the Euclidean metric. Please note that the convergence results can be deduced from Báez-Sánchez et al. (2012) using some simple inequalities between the Hausdorff and Euclidean metrics. However, we will use a direct approach which will give us precise estimations for the error of approximation. Clearly, $N_{\alpha}^{n}(A)$ preserves the support and core of $A$. Since $N_{\boldsymbol{\alpha}_{n}}^{n}(A) \in \mathbb{F}^{\pi_{n}(\boldsymbol{\alpha})}(\mathbb{R})$ for any $n \in \mathbb{N}$, let us find an estimation for $d\left(N_{\boldsymbol{\alpha}_{n}}^{n}(A), A\right)$ for some $n \geq 1$. Let us choose arbitrary $\beta \in[0,1]$. Then there exists $i \in\{0, \ldots, n\}$ such that $\beta \in\left[\alpha_{n, i}, \alpha_{n, i+1}\right]$. If $\left(N_{\boldsymbol{\alpha}_{n}}^{n}(A)\right)_{L}(\beta) \geq A_{L}(\beta)$ then

$$
\begin{aligned}
& \left|\left(N_{\boldsymbol{\alpha}_{n}}^{n}(A)\right)_{L}(\beta)-A_{L}(\beta)\right|=\left(N_{\boldsymbol{\alpha}_{n}}^{n}(A)\right)_{L}(\beta)-A_{L}(\beta) \\
& \quad \leq\left(N_{\boldsymbol{\alpha}_{n}}^{n}(A)\right)_{L}\left(\alpha_{n, i+1}\right)-A_{L}\left(\alpha_{n, i}\right)=A_{L}\left(\alpha_{n, i+1}\right)-A_{L}\left(\alpha_{n, i}\right) .
\end{aligned}
$$

On the other hand, if $\left(N_{\boldsymbol{\alpha}_{n}}^{n}(A)\right)_{L}(\beta)<A_{L}(\beta)$ then

$$
\begin{aligned}
\left|\left(N_{\boldsymbol{\alpha}_{n}}^{n}(A)\right)_{L}(\beta)-A_{L}(\beta)\right| & =A_{L}(\beta)-\left(N_{\boldsymbol{\alpha}_{n}}^{n}(A)\right)_{L}(\beta) \\
& \leq A_{L}\left(\alpha_{n, i+1}\right)-\left(N_{\boldsymbol{\alpha}_{n}}^{n}(A)\right)_{L}\left(\alpha_{n, i}\right) \\
& =A_{L}\left(\alpha_{n, i+1}\right)-A_{L}\left(\alpha_{n, i}\right)
\end{aligned}
$$

and therefore from the two inequalities we get

$\left|\left(N_{\boldsymbol{\alpha}_{n}}^{n}(A)\right)_{L}(\beta)-A_{L}(\beta)\right| \leq\left|A_{L}\left(\alpha_{n, i+1}\right)-A_{L}\left(\alpha_{n, i}\right)\right|$.

By similar reasonings, we obtain

$\left|\left(N_{\alpha_{n}}^{n}(A)\right)_{U}(\beta)-A_{U}(\beta)\right| \leq\left|A_{U}\left(\alpha_{n, i+1}\right)-A_{U}\left(\alpha_{n, i}\right)\right|$.

This implies that in general, for $\beta \in[0,1]$, we have

$$
\begin{aligned}
& \left|\left(N_{\boldsymbol{\alpha}_{n}}^{n}(A)\right)_{L}(\beta)-A_{L}(\beta)\right| \\
& \quad \leq \max _{i=0, \ldots, n}\left|A_{L}\left(\alpha_{n, i+1}\right)-A_{L}\left(\alpha_{n, i}\right)\right|, \\
& \quad\left|\left(N_{\boldsymbol{\alpha}_{n}}^{n}(A)\right)_{U}(\beta)-A_{U}(\beta)\right| \\
& \quad \leq \max _{i=0, \ldots, n}\left|A_{U}\left(\alpha_{n, i+1}\right)-A_{U}\left(\alpha_{n, i}\right)\right| .
\end{aligned}
$$

Theorem 4 Let us consider a sequence $\left(\boldsymbol{\alpha}_{n}\right)_{n=1,2, \ldots}, \boldsymbol{\alpha}_{n} \in$ $\mathfrak{A}_{n}$, where $\boldsymbol{\alpha}_{n}=\left(\alpha_{n, i}\right)_{i=0, \ldots, n+1}$ for all $n$, and such that $\left\|\boldsymbol{\alpha}_{n}\right\| \rightarrow 0$. If $A$ is a fuzzy number with continuous side functions then $\lim _{n \rightarrow \infty}^{(d)} \Pi_{\alpha_{n}}^{n}(A)=A$. Moreover, the rate of convergence is given by the inequalities

$$
\begin{aligned}
& d\left(A, \Pi_{\alpha_{n}}^{n}(A)\right) \leq \sqrt{2} \max _{i=0, \ldots, n}\left(\operatorname { m a x } \left\{\left|A_{L}\left(\alpha_{n, i+1}\right)-A_{L}\left(\alpha_{n, i}\right)\right|,\right.\right. \\
&\left.\left.\left|A_{U}\left(\alpha_{n, i+1}\right)-A_{U}\left(\alpha_{n, i}\right)\right|\right\}\right) .
\end{aligned}
$$

where $n \geq 1$.

Proof We will use in our proof the naïve approximator of $A$, introduced just above. Since functions $\left(N_{\boldsymbol{\alpha}_{n}}^{n}(A)\right)_{L}$, $\left(N_{\boldsymbol{\alpha}_{n}}^{n}(A)\right)_{U}, A_{L}$ and $A_{U}$ are all continuous, by the mean value theorem for integrals there exists $\xi_{n} \in[0,1]$ such that

$$
\begin{aligned}
d^{2}\left(N_{\boldsymbol{\alpha}_{n}}^{n}(A), A\right)= & \left(\left(N_{\boldsymbol{\alpha}_{n}}^{n}(A)\right)_{L}\left(\xi_{n}\right)-A_{L}\left(\xi_{n}\right)\right)^{2} \\
& +\left(\left(N_{\boldsymbol{\alpha}_{n}}^{n}(A)\right)_{U}\left(\xi_{n}\right)-A_{U}\left(\xi_{n}\right)\right)^{2},
\end{aligned}
$$

which by relations (9)-(10) implies (for any $n \geq 1$ )

$$
\begin{gathered}
d\left(N_{\boldsymbol{\alpha}_{n}}^{n}(A), A\right) \leq \sqrt{2} \max _{i=0, \ldots, n}\left(\operatorname { m a x } \left\{\left|A_{L}\left(\alpha_{n, i+1}\right)-A_{L}\left(\alpha_{n, i}\right)\right|,\right.\right. \\
\left.\left.\left|A_{U}\left(\alpha_{n, i+1}\right)-A_{U}\left(\alpha_{n, i}\right)\right|\right\}\right) .
\end{gathered}
$$


Since $A_{L}$ and $A_{U}$ are continuous and hence uniformly continuous and $\left\|\boldsymbol{\alpha}_{n}\right\| \rightarrow 0$, from the above inequalities it easily results $d\left(N_{\boldsymbol{\alpha}_{n}}^{n}(A), A\right) \rightarrow 0$.

Now let us prove that $\lim _{n \rightarrow \infty}^{(d)} \Pi_{\boldsymbol{\alpha}_{n}}^{n}(A)=A$. Since $N_{\boldsymbol{\alpha}_{n}}^{n}(A) \in \mathbb{F}^{\pi_{n}(\boldsymbol{\alpha})}(\mathbb{R})$ for any $n \in \mathbb{N}$ thus, by the definition of $\Pi_{\alpha_{n}}^{n}(A)$, it follows that

$d\left(A, \Pi_{\boldsymbol{\alpha}_{n}}^{n}(A)\right) \leq d\left(A, N_{\boldsymbol{\alpha}_{n}}^{n}(A)\right)$

and as $d\left(N_{\boldsymbol{\alpha}_{n}}^{n}(A), A\right) \rightarrow 0$, it is immediate now that $\lim _{n \rightarrow \infty}^{(d)} \Pi_{\boldsymbol{\alpha}_{n}}^{n}(A)=A$ with the convergence rate given by (11).

Recall that if $\left(X, d_{1}\right)$ and $\left(Y, d_{2}\right)$ are metric spaces then a function $f: X \rightarrow Y$ is called lipschitzian, if there exists a real positive constant $C$ such that

$d_{2}\left(f\left(x^{\prime}\right), f\left(x^{\prime \prime}\right)\right) \leq C d_{1}\left(x^{\prime}, x^{\prime \prime}\right)$,

for all $x^{\prime}, x^{\prime \prime} \in X$. It is well known that Lipschitz functions are continuous (thus, the property (iv) in Theorem 3 also implies the operator's continuity).

Proposition 1 Let us consider a sequence $\left(\boldsymbol{\alpha}_{n}\right)_{n=1,2, \ldots}, \boldsymbol{\alpha}_{n} \in$ $\mathfrak{A}_{n}$, where $\boldsymbol{\alpha}_{n}=\left(\boldsymbol{\alpha}_{n, i}\right)_{i=0, \ldots, n+1}$. If A denotes a fuzzy number such that $A_{L}$ and $A_{U}$ are Lipschitz functions with Lipschitz constants $C_{1}$ and $C_{2}$, respectively, then we have

$d\left(A, \Pi_{\boldsymbol{\alpha}_{n}}^{n}(A)\right) \leq C\left\|\boldsymbol{\alpha}_{n}\right\|$,

where $C=\sqrt{2} \max \left\{C_{1}, C_{2}\right\}$ and $n \in \mathbb{N}$.

Proof The hypothesis implies that

$\left|A_{L}\left(\alpha_{n, i}\right)-A_{L}\left(\alpha_{n, i-1}\right)\right| \leq C_{1}\left\|\boldsymbol{\alpha}_{n}\right\|$

$\left|A_{U}\left(\alpha_{n, i}\right)-A_{U}\left(\alpha_{n, i-1}\right)\right| \leq C_{2}\left\|\boldsymbol{\alpha}_{n}\right\|$.

for $i \in\{1, \ldots n+1\}$, which leads to the desired conclusion.

\subsection{Equidistant knots}

From the above theorem, we easily get some important propositions for the case of equidistant knots.

Proposition 2 Let us consider a sequence $\left(\boldsymbol{\alpha}_{n}\right)_{n=1,2, \ldots}$, where $\boldsymbol{\alpha}_{n} \in \mathfrak{A}_{n}, \alpha_{n, i}=\frac{i}{n+1}$ for all $i=0, \ldots, n+1$. If $A$ denotes a fuzzy number with continuous sides, then for all $n \in \mathbb{N}$ we have

$$
\begin{gathered}
d\left(A, \Pi_{\boldsymbol{\alpha}_{n}}^{n}(A)\right) \leq \sqrt{2} \max _{i=0, \ldots, n}\left(\operatorname { m a x } \left\{\left|A_{L}\left(\frac{i+1}{n+1}\right)-A_{L}\left(\frac{i}{n+1}\right)\right|,\right.\right. \\
\\
\left.\left.\left|A_{U}\left(\frac{i+1}{n+1}\right)-A_{U}\left(\frac{i}{n+1}\right)\right|\right\}\right) .
\end{gathered}
$$

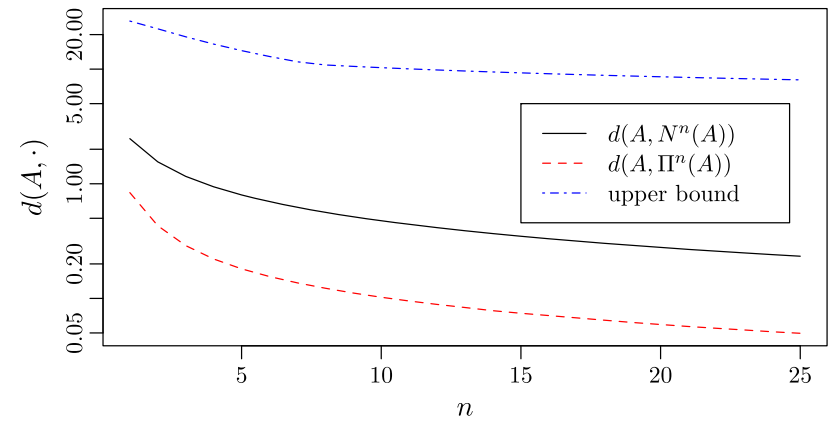

Fig. 2 Distance functions from Example 1. Note the logarithmic scale on the $y$ axis

Proof The proof is immediate by Theorem 4 and noting that $\alpha_{n, i}=\frac{i}{n+1}$ for every $i \in\{0,1, \ldots n+1\}$.

Example 1 Consider a fuzzy number $A$ with $\operatorname{supp} A=$ $[-5,20]$, core $A=[3,6]$ and $\alpha$-cuts given by

$A_{\alpha}=\left[-5+8\right.$ qbeta $\left.(\alpha ; 0.4,3), 6+14(1-\alpha)^{4}\right]$,

where qbeta $(\alpha ; a, b)$ denotes the quantile function of the Beta distribution $\mathrm{B}(a, b)$, i.e., the inverse of $F(x)=$ $\int_{0}^{x} t^{(a-1)}(1-t)^{(b-1)} \mathrm{d} t / \beta(a, b)$, where $\beta(a, b)$ denotes the Euler beta function.

In Fig. 2, we show the distance between $A$ and the naïve approximator, best-Euclidean approximator, and the theoretical upper bound given in Proposition 2, expressed as functions of $n$. We see that the best-Euclidean one has the fastest convergence rate. It is worth noting that the situation illustrated in Fig. 2 is not unique but it shows a typical behavior observed while studying many numerical examples.

Corollary 1 Let us consider a sequence $\left(\boldsymbol{\alpha}_{n}\right)_{n \geq 1}, \boldsymbol{\alpha}_{n} \in \mathfrak{A}_{n}$, where $\boldsymbol{\alpha}_{n}=(i /(n+1))_{i=0, \ldots, n+1}$. If A denotes a fuzzy number such that $A_{L}$ and $A_{U}$ are Lipschitz functions with Lipschitz constants $C_{1}$ and $C_{2}$, respectively, then we have

$d\left(A, \Pi_{\boldsymbol{\alpha}_{n}}^{n}(A)\right) \leq C /(n+1), \quad n \in \mathbb{N}$,

where $C=\sqrt{2} \max \left\{C_{1}, C_{2}\right\}$.

Proof The proof is immediate by applying Proposition 1 and noting that $\left\|\boldsymbol{\alpha}_{n}\right\|=1 /(n+1)$ for any $n \in \mathbb{N}, n \geq 1$.

Thus, we may conclude that in case of the fuzzy number approximation using equidistant knots the convergence rate is at most linear.

\subsection{Convergence w.r.t. some important characteristics}

Another important consequence of Theorem 4 is the convergence with respect to some important characteristics of a 
fuzzy number such as its value, ambiguity, expected interval or expected value. Let us recall briefly their definitions.

The value of a fuzzy number $A$ with respect to a nondecreasing function $c:[0,1] \rightarrow[0,1]$, called reducing function, where usually $c(0)=0$ and $c(1)=1$, is

$\operatorname{Val}_{c}(A)=\int_{0}^{1} c(\beta)\left(A_{L}(\beta)+A_{U}(\beta)\right) \mathrm{d} \beta$,

and might be seen as a typical value of the magnitude represented by the fuzzy number $A$. The next index, called the ambiguity, is given by

$\operatorname{Amb}_{c}(A)=\int_{0}^{1} c(\beta)\left(A_{U}(\beta)-A_{L}(\beta)\right) \mathrm{d} \beta$,

and characterizes the vagueness of $A$. These two characteristics were proposed for the first time by Delgado et al. (1988) to simplify the representation of fuzzy numbers.

The expected interval of a fuzzy number $A$ is given by (see Dubois and Prade 1987)

$\mathrm{EI}(A)=\left[\int_{0}^{1} A_{L}(\beta) \mathrm{d} \beta, \int_{0}^{1} A_{U}(\beta) \mathrm{d} \beta\right]$

while the expected value of $A$ is just the middle of its expected interval, i.e.,

$\mathrm{EV}(A)=\frac{1}{2}\left(\int_{0}^{1} A_{L}(\beta) \mathrm{d} \beta+\int_{0}^{1} A_{U}(\beta) \mathrm{d} \beta\right)$.

It is worth noting that the invariance of the above-mentioned characteristics applies a key role in the fuzzy number approximation (see Grzegorzewski and Mrówka 2005). Hence, the following result is of interest.

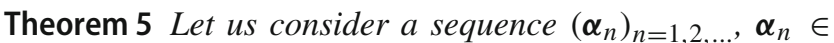
$\mathfrak{A}_{n}$, where $\boldsymbol{\alpha}_{n}=\left(\alpha_{n, i}\right)_{i=0, \ldots, n+1}$ for all $n$, and such that $\left\|\boldsymbol{\alpha}_{n}\right\| \rightarrow 0$. If A denotes a fuzzy number with continuous side functions and $c:[0,1] \rightarrow[0,1]$ is a continuous reducing function, then we have

$\lim _{n \rightarrow \infty} \operatorname{Val}_{c}\left(\Pi_{\alpha_{n}}^{n}(A)\right)=\operatorname{Val}_{c}(A)$,

$\lim _{n \rightarrow \infty} \operatorname{Amb}_{c}\left(\Pi_{\alpha_{n}}^{n}(A)\right)=\operatorname{Amb}_{c}(A)$,

$\lim _{n \rightarrow \infty} \operatorname{EI}\left(\Pi_{\alpha_{n}}^{n}(A)\right)=\operatorname{EI}(A)$,

$\lim _{n \rightarrow \infty} \operatorname{EV}\left(\Pi_{\boldsymbol{\alpha}_{n}}^{n}(A)\right)=\operatorname{EV}(A)$.

Proof Noting that $0 \leq c \leq 1$ and making use of the Schwarz inequality, we get

$$
\begin{aligned}
& \left|\int_{0}^{1} c(\beta) A_{L}(\beta) \mathrm{d} \beta-\int_{0}^{1} c(\beta)\left(\Pi_{\boldsymbol{\alpha}_{n}}^{n}(A)\right)_{L}(\beta)\right| \\
& \quad \leq \int_{0}^{1}\left|A_{L}(\beta)-\left(\Pi_{\alpha_{n}}^{n}(A)\right)_{L}(\beta)\right| \mathrm{d} \beta \\
& \quad \leq\left(\int_{0}^{1}\left(A_{L}(\beta)-\left(\Pi_{\alpha_{n}}^{n}(A)\right)_{L}(\beta)\right)^{2} \mathrm{~d} \beta\right)^{1 / 2} \\
& \quad \leq d\left(A, \Pi_{\boldsymbol{\alpha}_{n}}^{n}(A)\right) .
\end{aligned}
$$

Repeating the reasoning, we obtain

$$
\begin{aligned}
& \left|\int_{0}^{1} c(\beta) A_{U}(\beta) \mathrm{d} \beta-\int_{0}^{1} c(\beta)\left(\Pi_{\alpha_{n}}^{n}(A)\right)_{U}(\beta)\right| \\
& \quad \leq\left(\int_{0}^{1}\left(A_{U}(\beta)-\left(\Pi_{\alpha_{n}}^{n}(A)\right)_{U}(\beta)\right)^{2} \mathrm{~d} \beta\right)^{1 / 2} \\
& \quad \leq d\left(A, \Pi_{\alpha_{n}}^{n}(A)\right) .
\end{aligned}
$$

By the last two inequalities, we easily observe that

$$
\begin{aligned}
\max & \left\{\left|\operatorname{Amb}_{c}(A)-\operatorname{Amb}_{c}\left(\Pi_{\boldsymbol{\alpha}_{n}}^{n}(A)\right)\right|,\right. \\
& \left.\left|\operatorname{Val}_{c}(A)-\operatorname{Val}_{c}\left(\Pi_{\boldsymbol{\alpha}_{n}}^{n}(A)\right)\right|\right\} \leq 2 d\left(A, \Pi_{\boldsymbol{\alpha}_{n}}^{n}(A)\right) .
\end{aligned}
$$

which by Theorem 4 implies that (13) and (14) hold. Moreover, we have

$$
\begin{gathered}
\max \left\{\left|\operatorname{Amb}_{c}(A)-\operatorname{Amb}_{c}\left(\Pi_{\boldsymbol{\alpha}_{n}}^{n}(A)\right)\right|,\right. \\
\left.\left|\operatorname{Val}_{c}(A)-\operatorname{Val}_{c}\left(\Pi_{\boldsymbol{\alpha}_{n}}^{n}(A)\right)\right|\right\} \\
\leq 2 \sqrt{2} \max _{i=0, \ldots, n}\left(\operatorname { m a x } \left\{\left|A_{L}\left(\alpha_{n, i+1}\right)-A_{L}\left(\alpha_{n, i}\right)\right|,\right.\right. \\
\left.\left.\left|A_{U}\left(\alpha_{n, i+1}\right)-A_{U}\left(\alpha_{n, i}\right)\right|\right\}\right)
\end{gathered}
$$

for $n \geq 1$. Hence, we easily obtain the convergence of the expected interval and expected value, i.e., (15) and (16) hold, respectively.

\section{Computer implementation and applications}

In this section, we propose an algorithm to compute the nearest piecewise linear $n$-knot approximation. The reasonings are inspired by the particular case studied in Coroianu et al. (2013). Later on, we will show that best-Euclidean piecewise linear approximations are a better alternative than the classical naïve approximation in the implementation of fuzzy arithmetic on a computer.

\subsection{Algorithm}

Fix $\boldsymbol{\alpha}$. To perform the calculations, we should derive formulas for the elements $\phi_{i, j}=\left\langle\mathbf{e}_{i}, \mathbf{e}_{j}\right\rangle, i, j=1, \ldots, 2 n+4$, of the Gram matrix $\Phi=\left[\phi_{i, j}\right]$. It might be seen that we have: 


$$
\begin{array}{ll}
\phi_{1,1}=2, & \\
\phi_{i, j}=2-\left(\alpha_{j-1}+\alpha_{j-2}\right) / 2 & \text { for } i<j \leq n+2, \\
\phi_{j, j}=2-\left(2 \alpha_{j-1}+\alpha_{j-2}\right) / 3 & \text { for } j \leq n+2, \\
\phi_{i, n+3}=1 & \text { for } i \leq n+3, \\
\phi_{i, j}=\left(\alpha_{2 n-j+4}+\alpha_{2 n-j+5}\right) / 2 & \text { for } j \geq n+4, i<j \\
\phi_{j, j}=\left(2 \alpha_{2 n-j+4}+\alpha_{2 n-j+5}\right) / 3 & \text { for } j \geq n+4 .
\end{array}
$$

The $\Phi$ matrix is, of course, symmetric and-as already stated-invertible. Note that the elements above the diagonal do not depend on the row index $i$.

Fix $A$. Let $\mathbf{w}=\left(w_{1}, \ldots, w_{2 n+3}\right), \mathbf{w}^{\prime}=\left(w_{0}^{\prime}, \ldots, w_{2 n+3}^{\prime}\right)$ be such that:

$-w_{0}^{\prime}=0$

- For $i=1, \ldots, n+1$ do: $w_{i}:=\int_{\alpha_{i-1}}^{\alpha_{i}} A_{L}(\beta) d \beta$,

$$
w_{i}^{\prime}:=\frac{\int_{\alpha_{i-1}}^{\alpha_{i}} \beta A_{L}(\beta) d \beta-\alpha_{i-1} w_{i}}{\alpha_{i}-\alpha_{i-1}},
$$

$-w_{n+2}=w_{n+2}^{\prime}=0$,

- For $i=n+3, \ldots, 2 n+3$ do: $w_{i}:=\int_{\alpha_{2 n-i+3}}^{\alpha_{2 n-i+4}} A_{U}(\beta) d \beta$,

$$
w_{i}^{\prime}:=\frac{\alpha_{2 n-i+4} w_{i}-\int_{\alpha_{2 n-i+3}}^{\alpha_{2 n-i+4}} \beta A_{U}(\beta) d \beta}{\alpha_{2 n-i+4}-\alpha_{2 n-i+3}} .
$$

It may be shown easily that $\mathbf{b}=\left(b_{1}, \ldots, b_{2 n+4}\right)$ such that $b_{i}=\left\langle A, \mathbf{e}_{i}\right\rangle$, is determined by $b_{1}:=\sum_{i=1}^{2 n+3} w_{i}$ and $b_{i}:=b_{i-1}-w_{i-2}^{\prime}-w_{i-1}+w_{i-1}^{\prime}$ for $i=2, \ldots$, $2 n+4$.

Moreover, if $A \geq 0$ (i.e., if $\inf \operatorname{supp} A \geq 0$ ), then $w_{i} \geq w_{i}^{\prime}$ and it follows $b_{1} \geq b_{2} \geq \cdots \geq b_{2 n+4} \geq 0$. Note that $\mathbf{b}$ contains the whole information about $A$ needed to solve our approximation problem. Moreover, it may be seen that for $n=1$ the above derivations for $\Phi$ and $\mathbf{b}$ are equivalent to those presented in our previous paper (Coroianu et al. 2013), and for $n=0$ with those obtained by Ban (2009a).

Please note that if the solution to the system of $2 n+4$ linear equations $\Phi \breve{\boldsymbol{\delta}}^{T}=\mathbf{b}^{T}$ is such that $\breve{\delta}_{2}, \ldots, \breve{\delta}_{2 n+4} \geq$ 0 , then the problem of determining the nearest piecewise linear approximation is immediate: we have $\delta^{*}=\breve{\delta}$.

Example 2 Consider a fuzzy number $A$ with support [1, 4], core $[2,3]$, and $\alpha$-cuts given by $A_{\alpha}=\left[1+\sqrt{\alpha}, 4-\alpha^{2}\right]$. Let us determine the best approximation of $A$ by a piecewise linear 3-knot fuzzy number with $\boldsymbol{\alpha}=(0.25,0.5,0.75)$. We have

$$
\begin{aligned}
\mathbf{w}= & \left(\frac{1}{3}, \frac{1+\sqrt{2}}{6}, \frac{3-2 \sqrt{2}+3 \sqrt{3}}{12},\right. \\
& \left.\frac{11-3 \sqrt{3}}{12}, 0, \frac{155}{192}, \frac{173}{192}, \frac{185}{192}, \frac{191}{192}\right), \\
\mathbf{w}^{\prime}= & \left(0, \frac{7}{40}, \frac{19+4 \sqrt{2}}{120}, \frac{15+16 \sqrt{2}-6 \sqrt{3}}{120},\right. \\
& \left.\frac{-11+12 \sqrt{3}}{40}, 0, \frac{317}{768}, \frac{117}{256}, \frac{373}{768}, \frac{383}{768}\right)
\end{aligned}
$$

and thus

$$
\begin{array}{r}
\mathbf{b =}\left(\frac{16}{3}, \frac{207}{40}, \frac{599-16 \sqrt{2}}{120}, \frac{565+16 \sqrt{2}-36 \sqrt{3}}{120},\right. \\
\left.\left.\quad \frac{407+36 \sqrt{3}}{120}, \frac{11}{3}, \frac{2513}{768}, \frac{1855}{768}, \frac{379}{256}, \frac{383}{768}\right)\right) .
\end{array}
$$

Solving $\Phi \boldsymbol{\delta}^{* T}=\mathbf{b}^{T}$ we get

$$
\begin{aligned}
\delta^{*}=( & \frac{145-84 \sqrt{2}+54 \sqrt{3}}{105}, \frac{6}{35}(-2+14 \sqrt{2}-9 \sqrt{3}), \\
& -\frac{2}{35}(38+70 \sqrt{2}-81 \sqrt{3}), \frac{80}{7}+4 \sqrt{2}-\frac{342 \sqrt{3}}{35}, \\
& -\frac{2}{35}(328+42 \sqrt{2}-225 \sqrt{3}), \frac{12833+896 \sqrt{2}-7488 \sqrt{3}}{1120}, \\
& \left.\frac{7}{16}, \frac{5}{16}, \frac{3}{16}, \frac{1}{16}\right) \geq 0,
\end{aligned}
$$

and hence we directly obtain the desired approximator.

Here is the R code that calculates this result numerically via the piecewiseLinearApproximation () method from the FuzzyNumbers Gagolewski (2015) package.

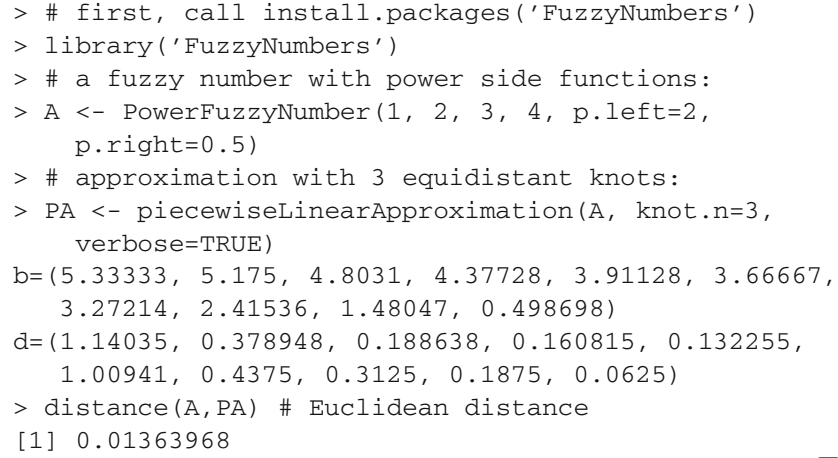

However, if $\breve{\delta}_{i}<0$ for some $i>1$, then we have to find the index set $K^{*} \subseteq\{2, \ldots, 2 n+4\}$ corresponding to the optimal solution. Intuitively, this set indicates between which knots (left or right) $\alpha$-cut bounds are constant functions. Generally, there are $2^{2 n+3}$ possible selections of the index sets and we know that at least one of them (as situation with $\delta_{k}^{*}=z_{k}^{*}=0$ 
is possible) leads to the solution fulfilling conditions from Theorem 2). Thus, although theoretically correct, in practice we cannot look for each possible $K$ and check whether it gives the desired result. Below we postulate an algorithm that finds the solution in up to $2 n+4$ steps.

First, please note that if $\mathrm{S}_{d}(\boldsymbol{\alpha}, \breve{\boldsymbol{\delta}}) \notin \mathbb{F}^{\pi_{n}(\boldsymbol{\alpha})}(\mathbb{R})$ then, by definition, we have $\left\langle A, \mathbf{e}_{i}\right\rangle=\left\langle\mathrm{S}_{d}(\boldsymbol{\alpha}, \breve{\boldsymbol{\delta}}), \mathbf{e}_{i}\right\rangle=b_{i}$ for all $i$. Thus, finding the best linear approximation of $A$ is the same as approximating the object $\mathrm{S}_{d}(\boldsymbol{\alpha}, \breve{\boldsymbol{\delta}})$ (corresponding to a pair of two square integrable piecewise linear functions).

Please note that it may be tempting to assume that as $n \rightarrow \infty$, then for all $\boldsymbol{\alpha}_{i} \in \mathfrak{A}_{i}$ such that $\boldsymbol{\alpha}_{i} \subset \boldsymbol{\alpha}_{i+1}$ we necessarily approach the solution with $z_{j, n}^{*}=0$ for all $j$. This is, unfortunately, not true, as a counterexample may easily be constructed (see Example 3).

It may be noted that if the conditions in Theorem 2 hold for a given index set $K^{*}$, then $\mathbf{z}^{*}$ and $\boldsymbol{\delta}^{*}$ may be obtained by solving the following system of $2 n+4$ linear equations for $\mathbf{x}$ :

$$
\begin{cases}\sum_{i=1}^{2 n+4}\left(\phi_{1, i} \mathbf{1}_{i \notin K^{*}}-\mathbf{1}_{i=1} \mathbf{1}_{i \in K^{*}}\right) x_{i}=b_{1} & \\ \vdots & \vdots \\ \sum_{i=1}^{2 n+4}\left(\phi_{2 n+4, i} \mathbf{1}_{i \notin K^{*}}-\mathbf{1}_{i=2 n+4} \mathbf{1}_{i \in K^{*}}\right) & x_{i}=b_{2 n+4}\end{cases}
$$

where $\mathbf{1}_{p}=1$ if $p$ is true and 0 if $p$ is false. This is done by setting $\delta_{i}^{*}=x_{i} \mathbf{1}_{i \notin K^{*}}$ and $z_{i}^{*}=x_{i} \mathbf{1}_{i \in K^{*}}$. The above system of equations may be written as

$\Phi^{\left(K^{*}\right)} \mathbf{x}^{T}=\mathbf{b}^{T}$,

where the $i$-th column of $\Phi^{\left(K^{*}\right)}$ is exactly the same as in $\Phi$ if $i \notin K^{*}$ and set to $\left(0, \ldots, 0, \phi_{i, i}^{\left(K^{*}\right)}=-1,0, \ldots, 0\right)^{T}$ otherwise. Moreover, $\Phi^{\left(K^{*}\right)}$ is always invertible.

The algorithm that finds the solution to the approximation problem of our interest is of "greedy" type. It relies on adding in each step to a temporary $K$ set such index $i>1$ at which an intermediate solution $x_{i}$ has the smallest negative value.

Heuristic Algorithm for determining best piecewise linear approximation of $A$ given $n$ and $\boldsymbol{\alpha}$ (cf. Coroianu et al. (2013):

1. Calculate $\Phi$ and $\mathbf{b}$ (according to $A_{L}, A_{U}, n$, and $\boldsymbol{\alpha}$ );

2. $K^{(1)}:=\emptyset$;

3. for $i=1,2, \ldots$ :

3.1. Solve $\Phi \mathbf{x}^{T}=\mathbf{b}^{T}$ for $\mathbf{x}$;

3.2. $m^{(i)}:=\min \left\{\arg \min _{i=2,3, \ldots, 2 n+4}\left\{x_{i}\right\}\right\}$;

3.3. if $\left(x_{m^{(i)}} \geq 0\right)$ :

3.3.1. $\boldsymbol{\delta}^{*}:=\left(x_{1}, x_{2} \mathbf{1}_{2 \notin K^{(i)}}, \ldots, x_{2 n+4} \mathbf{1}_{2 n+4 \notin K^{(i)}}\right)$;

3.3.2. return $\mathrm{S}_{d}\left(\boldsymbol{\alpha}, \boldsymbol{\delta}^{*}\right)$ as result and stop;

3.4. $\phi_{i, m}:=0$ for $i \neq m$;

3.5. $\phi_{m, m}:=-1$;

3.6. $K^{(i+1)}:=K^{(i)} \cup\left\{m^{(i)}\right\}$;
Note that $\mathrm{S}_{d}\left(\boldsymbol{\alpha}, \boldsymbol{\delta}^{*}\right)=\mathrm{S}\left(\boldsymbol{\alpha}, \operatorname{cumsum}\left(\boldsymbol{\delta}^{*}\right)\right)$, where $\operatorname{cumsum}\left(\boldsymbol{\delta}^{*}\right)=\left(\delta_{1}^{*}, \delta_{1}^{*}+\delta_{2}^{*}, \ldots, \delta_{1}^{*}+\delta_{2}^{*}+\cdots+\delta_{2 n+4}^{*}\right)$. Moreover, an example may be constructed easily for which at some step $m<0, m>\arg \min _{i=2,3, \ldots, 2 n+4}\left\{x_{i}\right\}$ and $m \notin K^{*}$.

Example 3 Consider a fuzzy number $A$ with support [1,3], core $\{2\}$, and $\alpha$-cuts given by $A_{\alpha}=\left[1+\alpha^{0.2}, 3-\alpha^{0.2}\right]$.

Let us determine the best approximation of $A$ by a piecewise linear 3-knot fuzzy number with $\boldsymbol{\alpha}=(0.75,0.8,0.9)$. The solution is obtained for $K=\{3,4,8,9\}$. Here is the output generated by the FuzzyNumbers Gagolewski (2015) package.

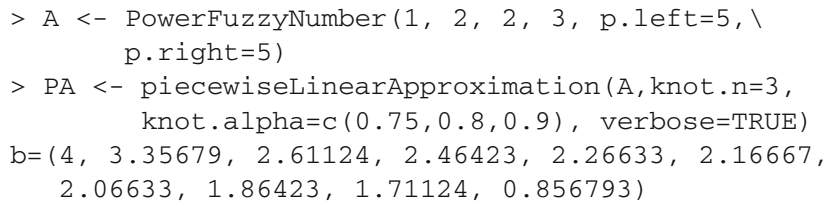

Please, note that adding more knots between 0.75 and 0.9 does not change the resulting piecewise linear fuzzy number (in the sense of $=$ ).

Remark 3 One can easily observe that the finding of the piecewise linear $n$-knot approximation of a fuzzy number using Theorem 2 directly may be represented as an 
instance of the Boolean satisfiability problem (SAT) which in general is NP-hard. The proposed heuristic algorithm drastically simplifies the process. Note that if it converges, the obtained solution is guaranteed to be optimal. Whether it always converges is still an open question. However, extensive simulation studies indicate that this is indeed the case.

Also note that from the computer processing perspective, calculating $\mathbf{b}$ is the most sensitive part of the procedure in terms of numeric error, due to the fact that $w_{i}$ and $w_{i}^{\prime}$ have to be obtained by some numerical quadratures, like the adaptive routine integrate ( ) in the $\mathrm{R}$ language. This requires the alpha-cut bounds to be well-behaving functions. Interestingly, the trapezoidal rule of integration (the Newton-Cotes formula of degree 1 , without subdivisions) will lead us to the naïve piecewise linear approximator (not optimal in general), in which we just probe the alpha-cut bounds at points in $\boldsymbol{\alpha}$.

\subsection{Computing on piecewise linear FNs}

Suppose we have a set of fuzzy numbers $\left\{A_{1}, \ldots, A_{k}\right\}$ and we would like to compute a series of operations on them, obtaining $B=f\left(A_{1}, \ldots, A_{k}\right)$. Let each operation be defined using the extension principle and rely on a proper transformations of their $\alpha$-cuts.

Most often such a task is performed numerically and not symbolically. Thus, the side functions of the fuzzy numbers must be discretized at a fixed, possibly large number of $\alpha$ cuts (see, e.g., Hanss 2005). Such a process involves nothing else than taking a naïve approximation of $A_{i}, i=1, \ldots, k$. Here, the values of membership functions at given $\alpha$-cuts (e.g., equidistant ones) are exact (of course, up to numeric error involved in calculating the operations). Of course, the larger the number of knots, the lower the computation speed but higher the accuracy.

In practice, we are interested in finding a trade-off between these two factors. An important question is whether, for fixed $n$ and $\boldsymbol{\alpha} \in \mathfrak{A}_{n}$, by considering the nearest-Euclidean approximation we will obtain results of higher quality than in case of a naïve approximation.

\section{a) Experiment setup:}

Let $N=N_{\alpha}^{n}\left(f\left(N_{\alpha}^{n}\left(A_{1}\right), \ldots, N_{\alpha}^{n}\left(A_{k}\right)\right)\right)$ and $\Pi=$ $\Pi_{\alpha}^{n}\left(f\left(\Pi_{\alpha}^{n}\left(A_{1}\right), \ldots, \Pi_{\alpha}^{n}\left(A_{k}\right)\right)\right)$ denote piecewise linear $n$ knot fuzzy numbers determined by taking, respectively, the naïve and nearest-Euclidean approximations of the input fuzzy numbers and then applying $f$ only at given $\boldsymbol{\alpha}$-cuts. Moreover, let $\Pi$-post $=\Pi_{\alpha}^{n}(B)$ denote the nearest-Euclidean piecewise linear approximation of the exact result, $B$. To verify this, let us perform a series of numerical experiments. First of all, one should be interested in:
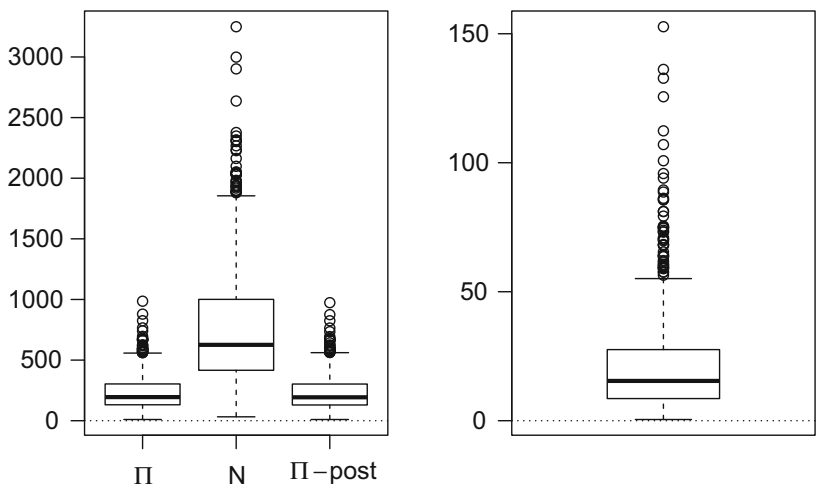

Fig. 3 Results of the numerical experiment: $d(B, \Pi), d(B, N)$, $d(B, \Pi$-post) (left subfigure) and $d(\Pi, \Pi$-post) (right subfigure)

- Comparing $d(B, N)$ and $d(B, \Pi)$ where $d$ denotes the Euclidean metric, i.e., determining whether it is better to use the nearest-Euclidean or the naïve approximation,

- Calculating $d(B, \Pi$-post $)$ and $d(\Pi, \Pi$-post), to determine a possible "error" which might appear if we perform computations directly on approximated fuzzy numbers.

To investigate these two issues, we performed a large number of computer simulations involving the following randomly generated types of fuzzy numbers:

- FNs with sides being power functions, i.e., $\alpha \mapsto \alpha^{p}$, where $p$ is uniformly distributed on the interval $(0,10)$, i.e., $p \sim U(0,10)$;

- FNs with sides defined via quantile functions of a beta distribution, $\alpha \mapsto$ qbeta $(\alpha, p, q), p, q \sim U(0.1,4)$.

Supports $\left[a_{1}, a_{4}\right]$ and cores $\left[a_{2}, a_{3}\right]$ were also generated by $\left(a_{1}, a_{2}, a_{3}, a_{4}\right):=\operatorname{sort}\left(a_{1}^{\prime}, a_{2}^{\prime}, a_{3}^{\prime}, a_{4}^{\prime}\right)$, with $a_{1}^{\prime}, a_{2}^{\prime}, a_{3}^{\prime}, a_{4}^{\prime}$ following the uniform distribution.

b) Distance between exact results and the results computed on approximated fuzzy numbers:

As all the considered scenarios exhibited the same patterns, below we will only present results for the scenario with $f\left(A_{1}, \ldots, A_{9}\right)=\left(A_{1}+A_{2}\right) A_{3}-A_{4}\left(A_{5}-A_{6}\right) \log \left(A_{7}\right)+$ $\exp \left(A_{8}\right) / 2^{A_{9}}$, with $M=1000$ Monte Carlo iterations, and $n=3$ equidistant knots. Figure 3 depicts the boxplots of $d(B, \Pi), d(B, N)$ and $d(B, \Pi$-post), as well as $d(\Pi, \Pi$-post). It is worth noting that $d(B, \Pi)<d(B, N)$ in each case. In other words, applying computations on nearestEuclidean approximated inputs leads to smaller errors (as measured by the Euclidean distance) than for the naïve approximations. Figure 4 depicts the difference between an exemplary exact output and ones that are computed using two types of piecewise linear approximations.

Next, Fig. 5 presents scatter plots for each pair of observations from $\{d(B, \Pi), d(B, N), d(B, \Pi$-post $)\}$. Interestingly, 


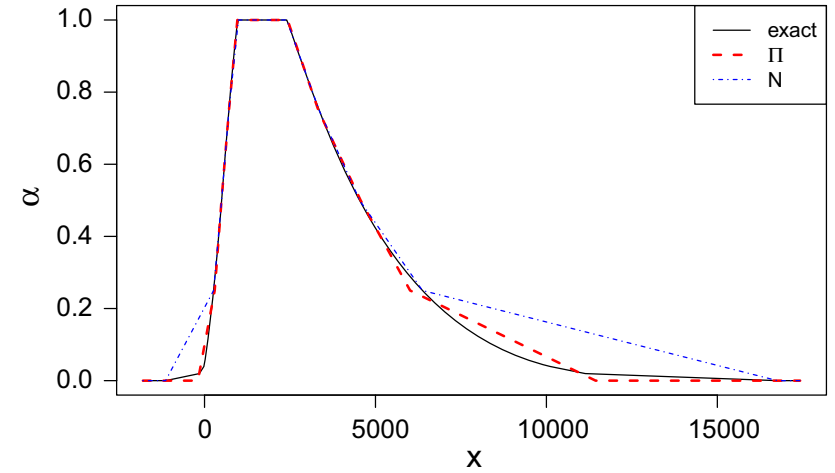

Fig. 4 An exemplary result of applying $f\left(A_{1}, \ldots, A_{9}\right)=\left(A_{1}+\right.$ $\left.A_{2}\right) A_{3}-A_{4}\left(A_{5}-A_{6}\right) \log \left(A_{7}\right)+\exp \left(A_{8}\right) / 2^{A_{9}}$ : the exact one, and ones computed on best-Euclidean and naïvely approximated inputs

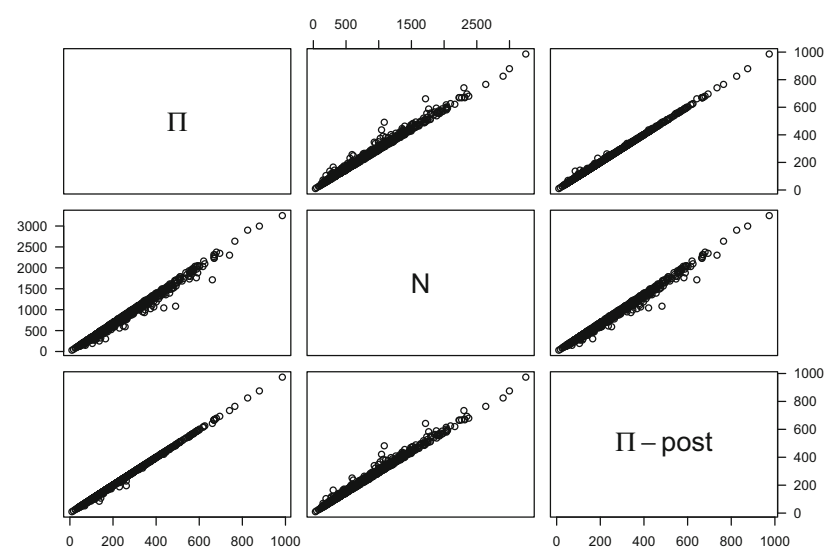

Fig. 5 Scatter plots for $d(B, \Pi), d(B, N)$, and $d(B, \Pi$-post)

we observe high linear association between the errors (Pearson's $r>0.99$ ). Applying linear regression, we get the following relationships for $n=3$ :

$d(B, \Pi) \simeq 1.00544 d(B, \Pi$-post $)$

$d(B, N) \simeq 3.31601 d(B, \Pi$-post $)$

$d(B, \Pi) \simeq 0.30237 d(B, N)$

which are quite nicely fitted $\left(R^{2}>0.99\right)$. Moreover, for $n=10$ equidistant knots we have

$$
\begin{aligned}
& d(B, \Pi) \simeq 1.00474 d(B, \Pi \text {-post }) \\
& d(B, N) \simeq 2.29118 d(B, \Pi \text {-post }) \\
& d(B, \Pi) \simeq 0.43851 d(B, N) .
\end{aligned}
$$

We see that the error induced by the nearest-Euclidean approximation is more than $2-3$ times smaller than that accompanying the naïve approximation.

Moreover, in Table 1 we list basic summary statistics of the $L_{2}$ error measure between the exact solution and ones obtained by means on acting on approximated fuzzy num-
Table 1 Basic summary statistics of the error measure between the solution to $f\left(A_{1}, \ldots, A_{9}\right)=\left(A_{1}+A_{2}\right) A_{3}-A_{4}\left(A_{5}-A_{6}\right) \log \left(A_{7}\right)+$ $\exp \left(A_{8}\right) / 2^{A_{9}}$ and one obtained by the two studied approximators for different $n(0-$ trapezoidal, 1,3 , and 10$)$

\begin{tabular}{lrrrrrr}
\hline & Min & Q1 & Med & Mean & Q3 & Max \\
\hline$d\left(B, \Pi^{0}\right)$ & 96 & 207 & 308 & 439 & 618 & 1129 \\
$d\left(B, \Pi^{1}\right)$ & 74 & 141 & 224 & 261 & 366 & 564 \\
$d\left(B, \Pi^{3}\right)$ & 48 & 91 & 138 & 150 & 192 & 283 \\
$d\left(B, \Pi^{10}\right)$ & 27 & 38 & 73 & 80 & 109 & 179 \\
$d\left(B, N^{0}\right)$ & 443 & 838 & 1184 & 1393 & 1755 & 2690 \\
$d\left(B, N^{1}\right)$ & 297 & 529 & 883 & 862 & 1081 & 1606 \\
$d\left(B, N^{3}\right)$ & 146 & 296 & 407 & 459 & 615 & 949 \\
$d\left(B, N^{10}\right)$ & 60 & 88 & 167 & 181 & 246 & 409 \\
\hline
\end{tabular}

Table 2 Basic characteristics of the empirical distribution of $F_{1}=d_{M}(\operatorname{supp} B, \operatorname{supp} \Pi), F_{2}=d_{M}(\operatorname{supp} B, \operatorname{supp} \Pi-$ post $), F_{3}=$ $d_{M}($ core $B$, core $\Pi)$ and $F_{4}=d_{M}($ core $B$, core $\Pi$-post $)$

\begin{tabular}{rrrrrrr}
\hline & Min & Q1 & Med & Mean & Q3 & Max \\
\hline$F_{1}$ & 30 & 960 & 1674 & 2095 & 2865 & 10296 \\
$F_{2}$ & 28 & 959 & 1677 & 2094 & 2864 & 10202 \\
$F_{3}$ & 2 & 151 & 347 & 560 & 716 & 5098 \\
$F_{4}$ & 1 & 152 & 349 & 557 & 719 & 4995 \\
\hline
\end{tabular}

bers. We see that with a large number of knots we get much more precise results and that the best $L_{2}$ approximator is far better than the naïve one.

c) Preservation of fuzzy numbers' characteristics:

We might be also interested whether the nearest-Euclidean approximation preserves better some important characteristics of the concerned fuzzy numbers. Of course, the support and core of $B$ and $N$ are the same. To measure the error for $\Pi$ and $\Pi$-post, we will use the Moore's interval metric Moore (1962), given by $d_{M}([a, b],[c, d])=\max \{|a-c|,|b-d|\}$.

Table 2 shows some basic summary statistics for those measures of interest. We observe again the almost perfect correlation $(r>0.999)$ between $d_{M}(\operatorname{supp} B, \operatorname{supp} \Pi)$ and $d_{M}\left(\operatorname{supp} B\right.$, supp $\Pi$-post) as well as between $d_{M}$ (core $B$, core $\Pi$ ) and $d_{M}$ (core $B$, core $\Pi$-post). Indeed

$d_{M}(\operatorname{supp} B, \operatorname{supp} \Pi) \simeq 1.000676 d_{M}(\operatorname{supp} B, \operatorname{supp} \Pi-$ post $)$ $d_{M}($ core $B$, core $\Pi) \simeq 1.007172 d_{M}($ core $B$, core $\Pi$-post $)$.

The above-mentioned results are very interesting especially that the stability of the support and core is very important in fuzzy number approximation (for more details we refer the reader to Coroianu et al. 2014a).

Lastly, let us investigate the difference between $B$ 's ambiguity, width, expected value, or value and the corresponding characteristics of $\Pi, N$, and $\Pi$-post. The relevant boxplots 

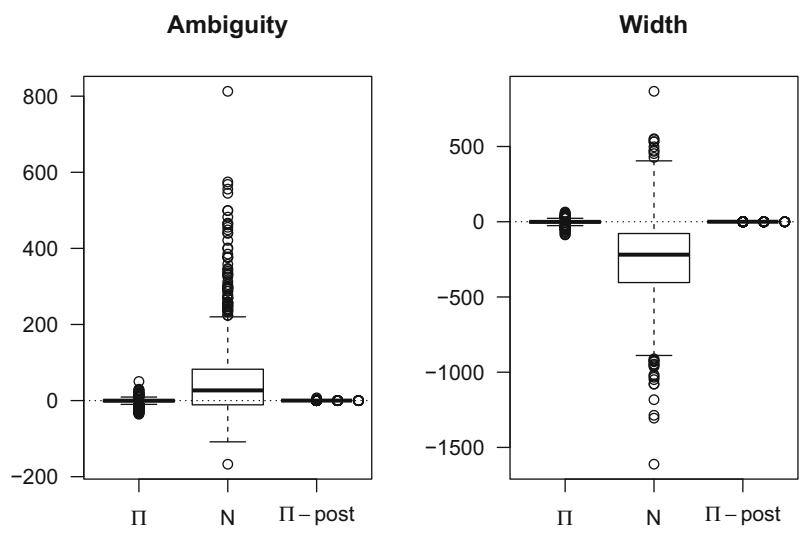

Fig. 6 amb $B-\operatorname{amb} \Pi$, amb $B-\operatorname{amb} N$, and amb $B-\operatorname{amb} \Pi$-post (left subfigure) as well as width $B-$ width $\Pi$, width $B-$ width $N$, and width $B$ - width $\Pi$-post (right subfigure)
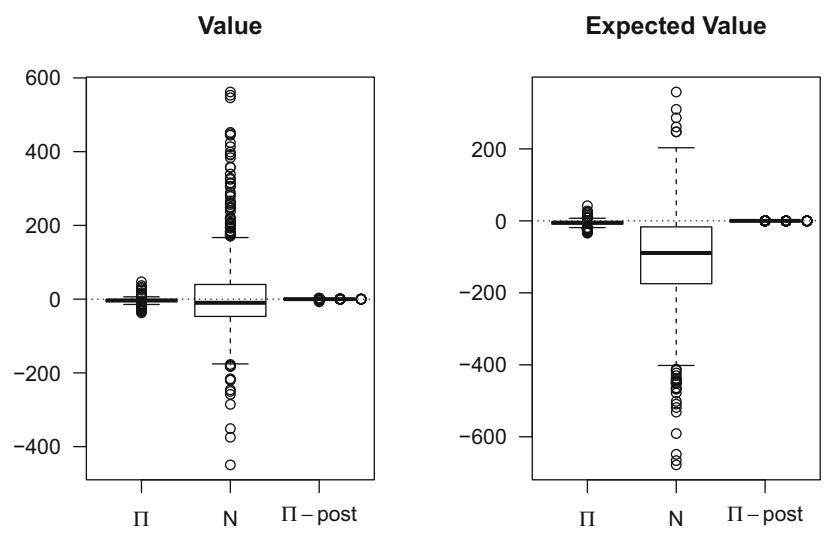

Fig. 7 val $B$ - val $\Pi$, val $B$ - val $N$, and val $B$ - val $\Pi$-post (left subfigure) as well as ev $B-\operatorname{ev} \Pi$, ev $B-\operatorname{ev} N$, and ev $B-$ ev $\Pi$-post (right subfigure)

are depicted in Figs. 6 and 7. We see that the nearestEuclidean approximation preserves these fuzzy numbers characteristics much better than the naïve approximation. Although these characteristics are not ideally preserved, they are reconstructed quite accurately.

\section{Conclusions}

The problem of fuzzy number approximation by the piecewise linear fuzzy numbers introduced in Báez-Sánchez et al. (2012) was considered. In this paper, the nearest piecewise linear approximation with respect to the Euclidean metric was concerned. The properties of the approximator, including the asymptotic ones, were investigated. The practical implementation of the approximation algorithm is available in the FuzzyNumbers package for R by Gagolewski (2015) .

The results on convergence indicate some advantages of the piecewise approximation. Let us recall that the general idea is not only to approximate an arbitrary fuzzy number by another fuzzy number with a simpler representation, but to find such an approximation which also possesses some interesting properties. For example, in the papers Ban (2008), Coroianu (2011), Grzegorzewski and Mrówka (2005, 2007), Yeh (2008a), the trapezoidal approximation preserving the expected interval is studied. Although this type of approximation is important in some applications, the expected interval invariance may imply that other important characteristics, such as the value or ambiguity, are not generally preserved there (see, e.g., Ban 2008). Additionally, in some cases as a result of the trapezoidal approximation we obtain a degenerated triangular fuzzy number such that the output support and core is relatively far from the input support and core, respectively. Similarly, the trapezoidal approximation preserving the ambiguity (see Ban and Coroianu 2012), trapezoidal approximation preserving the ambiguity and value (see Ban et al. 2011a) or the trapezoidal approximation preserving the core (see Abbasbandy and Hajjari 2009b), entails the loss of invariance of some other important characteristics of fuzzy numbers.

When discussing about our best-Euclidean piecewise linear approximator, one may ask naturally whether it is really better than the naïve approximator, i.e., an $n$-knot piecewise linear fuzzy number that interpolates both $A_{L}$ and $A_{U}$ at the knots. Although, as usually in such cases, there is no overall champion, it is worth stressing that

- In the case of the best-Euclidean approximator, we have a guarantee that the solution is the closest possible in terms of the $L_{2}$ metric, while there is no such a guarantee in the naïve case.

- The best-Euclidean approximator has faster rate of convergence than the naïve one. Convergence rate is determined w.r.t. $L_{2}$ metric, thus it is obvious that the suggested approximator will be better in this case.

- One can easily observe that the finding of the piecewise linear $n$-knot approximation of a fuzzy number using Theorem 2 directly may be represented as an instance of the Boolean satisfiability problem (SAT) which in general is NP-hard. The proposed heuristic algorithm drastically simplifies the process. Note that if it converges, the obtained solution is guaranteed to be optimal. Whether it always converges is still an open question. However, extensive simulation studies indicate that this is indeed the case.

- The algorithm proposed in Sect. 6 makes the computations as easy as in the naïve case (from the practitioner's perspective). However, practical computation on the bestEuclidean approximations are more exact (see simulation study), especially if the series of arithmetic operations are involved, while the naïve approximators lead to a great error propagation. 
Many interesting problems related to the approximation method suggested in this paper are still open. Firstly, a study of the global uniform convergence of the functions $\Pi_{\alpha_{\mathbf{n}}}(A)_{L}$ and $\Pi_{\alpha_{\mathbf{n}}}(A)_{U}$ which will imply the convergence with respect to the support and core is strongly desired.

Next important problem is a comparison between different methods that one can apply to approximate fuzzy numbers. In particular, it would be interesting to compare our general piecewise linear approximation and the approximation of fuzzy numbers by using the $F$-transform (see Coroianu and Stefanini 2016; Stefanini and Sorini 2012) or with approximation by the Bernstein operators of max-product kind (see Coroianu et al. 2014b). Here we can discuss many issues as, for instance, which method is easier to implement on the computer. Then, of course, we could compare their convergence rates (with respect to different kind of metrics such as the Chebyshev or the Euclidean), and their errors in approximating the important characteristics.

Finally, as it was mentioned above, the convergence of the algorithm proposed in Sect. 6 is still an open question.

Acknowledgements The contribution of Lucian Coroianu and Marek Gagolewski was co-founded by the European Union under the European Social Found. Project POKL "Information technologies: Research and their interdisciplinary applications", Agreement UDA-POKL.04.01.0100-051/10-00. The contribution of Lucian Coroianu was also supported by a grant of Ministry of Research and Innovation, CNCS-UEFISCDI, project number PN-III-P1-1.1-PD-2016-1416, within PNCDI III.

\section{Compliance with ethical standards}

Conflict of interest The authors declare that there is no conflict of interests regarding the publication of this paper.

Ethical approval This article does not contain any studies with human participants or animals performed by any of the authors.

Open Access This article is distributed under the terms of the Creative Commons Attribution 4.0 International License (http://creativecomm ons.org/licenses/by/4.0/), which permits unrestricted use, distribution, and reproduction in any medium, provided you give appropriate credit to the original author(s) and the source, provide a link to the Creative Commons license, and indicate if changes were made.

\section{References}

Abbasbandy S, Amirfakhrian M (2006) The nearest trapezoidal form of a generalized left right fuzzy number. J Approx Reason 43:166178

Abbasbandy S, Asady B (2004) The nearest trapezoidal fuzzy number to a fuzzy quantity. Appl Math Comput 156:381-386

Abbasbandy S, Hajjari T (2009a) A new approach for ranking of trapezoidal fuzzy numbers. Comput Math Appl 57:413-419

Abbasbandy S, Hajjari T (2009b) Weighted trapezoidal approximationpreserving cores of a fuzzy number. Comput Math Appl 59:30663077
Abbasbandy S, Ahmady E, Ahmady N (2010) Triangular approximations of fuzzy numbers using $\alpha$-weighted valuations. Soft Comput 14:71-79

Báez-Sánchez AD, Moretti AC, Rojas-Medar MA (2012) On polyghonal fuzzy sets and numbers. Fuzzy Sets Syst 209:54-65

Ban AI (2008) Approximation of fuzzy numbers by trapezoidal fuzzy numbers preserving the expected interval. Fuzzy Sets Syst 159:1327-1344

Ban AI (2009a) On the nearest parametric approximation of a fuzzy number-revisited. Fuzzy Sets Syst 160:3027-3047

Ban AI (2009b) Trapezoidal and triangular approximations of fuzzy numbers-inadvertences and corrections. Fuzzy Sets Syst 160:3048-3058

Ban AI (2011) Remarks and corrections to the triangular approximations of fuzzy numbers using $\alpha$-weighted valuations. Soft Comput $15: 351-361$

Ban AI, Ban O (2012) Optimization and extensions of a fuzzy multicriteria decision making method and applications to selection of touristic destinations. Expert Syst Appl 39:7216-7225

Ban AI, Coroianu L (2011) Discontinuity of the trapezoidal fuzzy number-valued operators preserving core. Comput Math Appl 62:3103-3110

Ban AI, Coroianu L (2012) Nearest interval, triangular and trapezoidal approximation of a fuzzy number preserving ambiguity. Int J Approx Reason 53:805-836

Ban AI, Coroianu L (2014) Existence, uniqueness and continuity of trapezoidal approximations of fuzzy numbers under a general condition. Fuzzy Sets Syst 257:3-22

Ban AI, Coroianu L (2015a) Existence, uniqueness, calculus and properties of triangular approximations of fuzzy numbers under a general condition. Int J Approx Reason 62:1-26

Ban AI, Coroianu L (2015b) Simplifying the search for effective ranking of fuzzy numbers. IEEE Trans Fuzzy Syst 23:327-339

Ban AI, Coroianu L (2016) Symmetric triangular approximations of fuzzy numbers under a general condition and properties. Soft Comput 20:1249-1261

Ban AI, Brândaş A, Coroianu L, Negruţiu C, Nica O (2011a) Approximations of fuzzy numbers by trapezoidal fuzzy numbers preserving the value and ambiguity. Comput Math Appl 61:1379-1401

Ban AI, Coroianu L, Grzegorzewski P (2011b) Trapezoidal approximation and aggregation. Fuzzy Sets Syst 177:45-59

Ban AI, Coroianu L, Grzegorzewski P (2015) Fuzzy numbers: approximations, ranking and applications. Institute of Computer Science, Polish Academy of Sciences, Warsaw

Ban AI, Coroianu L, Khastan A (2016) Trapezoidal approximation and aggregation. Fuzzy Sets Syst 283:56-82

Bodjanova S (2005) Median value and median interval of a fuzzy number. Inf Sci 172:73-89

Carlsson C, Fullér R (2011) Possibility for decision. Springer, Berlin

Chanas S (2001) On the interval approximation of a fuzzy number. Fuzzy Sets Syst 122:353-356

Coroianu L (2011) Best lipschitz constant of the trapezoidal approximation operator preserving the expected interval. Fuzzy Sets Syst 165:81-97

Coroianu L (2012) Lipschitz functions and fuzzy number approximations. Fuzzy Sets Syst 200:116-135

Coroianu L, Stefanini L (2016) General approximation of fuzzy numbers by f-transform. Fuzzy Sets Syst 288:46-74

Coroianu L, Gagolewski M, Grzegorzewski P (2013) Nearest piecewise linear approximation of fuzzy numbers. Fuzzy Sets Syst 233:2651

Coroianu L, Gagolewski M, Grzegorzewski P, Firozja MA, Houlari T (2014a) Piecewise linear approximation of fuzzy numbers preserving the support and core. In: Laurent A, et al (eds) Proceedings of the 15th international conference IPMU 2014. CCIS, Springer, vol 443, pp 244-253 
Coroianu L, Gal SG, Bede B (2014b) Approximation of fuzzy numbers by bernstein operators of max-product kind. Fuzzy Sets Syst 257:41-66

Delgado M, Vila MA, Voxman W (1988) On a canonical representation of a fuzzy number. Fuzzy Sets Syst 93:125-135

Diamond P, Kloeden P (1994) Metric spaces of fuzzy sets. Theory and applications. World Scientific, Singapore

Dubois D, Prade H (1987) The mean value of a fuzzy number. Fuzzy Sets Syst 24:279-300

Facchinetti G, Ricci RG (2004) A characterization of a general class of ranking functions on triangular fuzzy numbers. Fuzzy Sets Syst 146:297-312

Gagolewski M (2015) Fuzzynumbers package: tools to deal with fuzzy numbers in R. http://FuzzyNumbers.rexamine.com

Grzegorzewski P (1998) Metrics and orders in space of fuzzy numbers. Fuzzy Sets Syst 97:83-94

Grzegorzewski P (2002) Nearest interval approximation of a fuzzy number. Fuzzy Sets Syst 130:321-330

Grzegorzewski P (2008a) New algorithms for trapezoidal approximations of fuzzy numbers preserving the expected interval. In: Magdalena L, et al. (eds) Proceedings of the 12th international conference IPMU 2008. CCIS, Springer, vol 299, pp 117-123

Grzegorzewski P (2008b) Trapezoidal approximations of fuzzy numbers preserving the expected interval-algorithms and properties. Fuzzy Sets Syst 47:1354-1364

Grzegorzewski P (2010) Algorithms for trapezoidal approximations of fuzzy numbers preserving the expected interval. In: BouchonMeunier B et al (eds) Foundations of reasoning under uncertainty. Springer, Berlin, pp 85-98

Grzegorzewski P (2012) On the interval approximation of fuzzy numbers. In: Greco $S$, et al (eds) Proceedings of the 14th international conference IPMU 2012. CCIS, Springer, vol 299, pp 58-68

Grzegorzewski P (2013) Fuzzy number approximation via shadowed sets. Inf Sci 225:35-46

Grzegorzewski P, Mrówka E (2005) Trapezoidal approximations of fuzzy numbers. Fuzzy Sets Syst 153:115-135

Grzegorzewski P, Mrówka E (2007) Trapezoidal approximations of fuzzy numbers-revisited. Fuzzy Sets Syst 158:757-768
Grzegorzewski P, Pasternak-Winiarska K (2009) Bi-symmetrically weighted trapezoidal approximations of fuzzy numbers. In: Proceedings of ninth international conference on intelligent systems design and applications ISDA'09. IEEE, pp 318-323

Grzegorzewski P, Pasternak-Winiarska K (2014) Natural trapezoidal approximations of fuzzy numbers. Fuzzy Sets Syst 250:90-109

Hanss M (2005) Applied fuzzy arithmetic. Springer, Berlin

Hong DH, Hwang C (1997) A T-sum bound of LR-fuzzy numbers. Fuzzy Sets Syst 91:239-252

Kolesárová A (1995) Triangular norm-based addition of linear fuzzy numbers. Tatra Mt Math Publ 6:75-82

Moore RE (1962) Interval arithmetic and automatic error analysis in digital computing. Tech Rep 25, Department of Mathematics Stanford University

Nasibov NE, Peker S (2008) On the nearest parametric approximation of a fuzzy number. Fuzzy Sets Syst 159:1365-1375

Stefanini L, Sorini L (2012) Approximation of fuzzy numbers by f-transform. In: Greco S, et al (eds) Proceedings of the 14th international conference IPMU 2012. CCIS, Springer, vol 299, pp 69-78

Yeh CT (2007) A note on trapezoidal approximations of fuzzy numbers. Fuzzy Sets Syst 158:747-754

Yeh CT (2008a) On improving trapezoidal and triangular approximations of fuzzy numbers. J Approx Reason 48:297-313

Yeh CT (2008b) Trapezoidal and triangular approximations preserving the expected interval. Fuzzy Sets Syst 159:1345-1353

Yeh CT (2009) Weighted trapezoidal and triangular approximations of fuzzy numbers. Fuzzy Sets Syst 160:3059-3079

Yeh CT (2011) Weighted semi-trapezoidal approximations of fuzzy numbers. Fuzzy Sets Syst 165:61-80

Yeh CT (2017) Note on symmetric triangular approximations of fuzzy numbers under a general condition and properties. Soft Comput 11:27-47

Yeh CT, Chu HM (2011) Approximations by LR-fuzzy numbers. Fuzzy Sets Syst 257:23-40

Publisher's Note Springer Nature remains neutral with regard to jurisdictional claims in published maps and institutional affiliations. 